\title{
Three-dimensional description of the spontaneous onset of homochirality on the surface of a conglomerate crystal phase. Supporting information
}

\author{
Raphaël Plasson, Kouichi Asakura, Dilip K. Kondepudi
}

\section{Description of the Program}

\subsection{Algorithm}

A discrete stochastic description of the system has been chosen ${ }^{1}$. Each elementary growth subunit is supposed attached to one element of a three-dimensional matrix, and no diffusion is supposed to occur (this is actually not a real limitation, as solid compounds can't diffuse, and as all liquid compounds are identical). All compounds that can react at a given time $t$ are listed. They can either be a L subunit (liquid subunit, being on top of an interfacial (I) crystalline subunit) or G compounds (growth layer subunit, waiting to become part of the crystal (C) phase). The probability of each one of these subunits to be transformed in another one is calculated on the basis of the corresponding stochastic kinetic rates, and of their respective environment: the four lateral subunit (which can catalyze and induce crystallizations, red block in figure S1) and the underneath subunit (acting as a fixation site, blue block in figure S1.

The total sum of all stochastic rates of all possible events $\delta p$ is calculated, and a random number is drawn from the uniformly distributed interval $\left[0, \delta p 2^{2}\right.$. This number allows to choose which event will actually happen in the infinitesimal time $\delta t=\frac{1}{\delta p}$.

The interest of such an algorithm is to inherently take into account the statistical fluctuations 3 and to keep the microscopic (molecular) description. On top of that, all stochastic rates are in the same unit $\left(s^{-1}\right)$ - as representing directly a probability of reaction rather than a macroscopic kinetic rates — which maker easier the comparison between all kinetic rates (which can't be directly done in a macroscopic description, as $k_{01}, k_{02}$ and $k_{20}$ are first order rates, while $k_{11} k_{12}$ and $k_{12}$ are second order reactions)

\subsection{Implementation}

The program was written in $\mathrm{C}++_{+}$, so that an objectoriented structure can guarantee an easy maintenance and extensibility of the source code, and a clear organization of data and processes. The core program is handled by a batch program written in Python 5 in order to have access to high level function for automatic plotting of functions (using Gnuplot 6 ) and the generation of graphical reports (using PII 7 ) for each calculation.

\subsubsection{Classes}

The program was organized in six compounds:

Medium class This class contain the 3-D matrix describing the growing crystal, and its link (via Layer and Sublayer classes) with possible reactions (described in event class). It allows the initiation of calculation for a given number of elementary reaction.

Layer class Description of the growth front, divided by sublayers (each sublayer corresponds to one column of the total growth front).

Sublayer class One column of the Layer class, divided in a list of Events. This class and the preceding one are the link between Medium and Event class. An initially more general Layer class have been splitted into these two Layer and Sublayer classes for calculation time optimization purpose. They permit to choose from the Medium class which possible reaction (one instance of Reac class) will occur (see the paragraph 1.2 .2 for more details).

${ }^{1}$ J. J. Lukkien, J. P. L. Segers, and P. A. J. Hilbers. Efficient Monte-Carlo methods for the simulation of catalytic surface reactions Phys. Rev. E, 58(2):2598-2613, 1998.

${ }^{2}$ double precision uniformly distributed random number, calculated by the drand48 function from standard C library

${ }^{3}$ see section 2 for more informations about statistical fluctuations

${ }^{4}$ see section 3 for the relationship between stochastic and macroscopic kinetic rates 

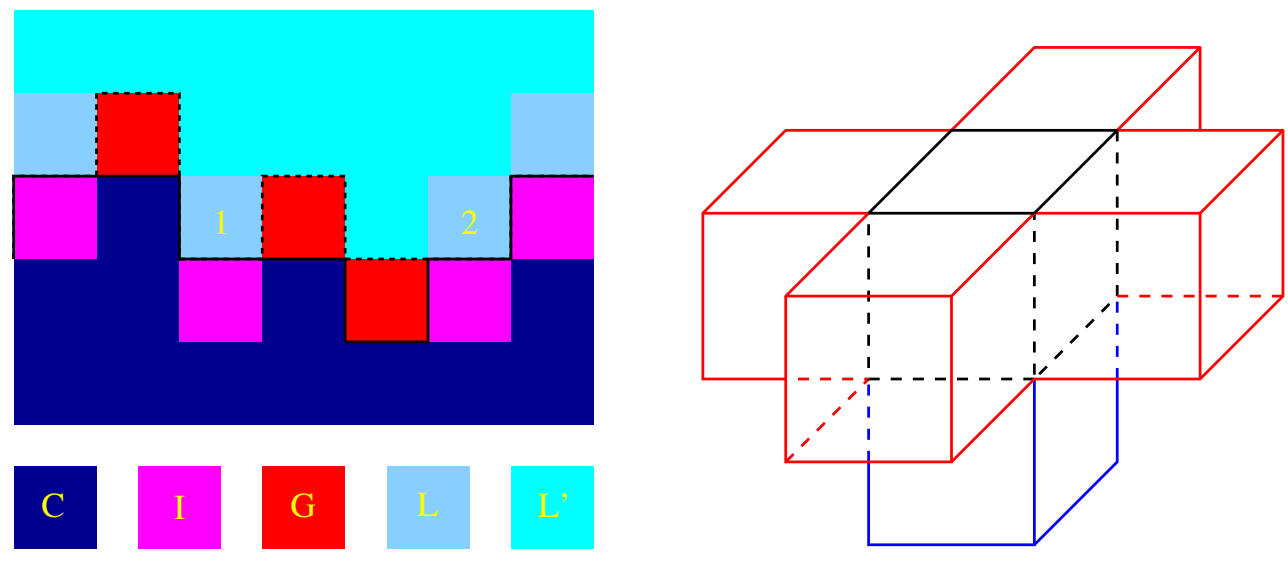

Figure S1: Schematic description of a growing layer. C: crystal bulk phase, I: Interface of crystal to liquid, G: growth layer, L: liquid phase that can react, L: liquid phase that is not in contact with the interface. 3D representation: black, center block; red, lateral block; blue, underneath block.

Event class This class instantiate the several possible reactions than can occur in the system, and can perform them when asked through the Medium->Layer->Sublayer chain.

Reac structure This structure gives all the informations relative to a given reaction (coordinates, kinetic rates, type of reaction, and the kind of compound to be generated)

Linked_node class Intermediate class only used by Medium for the 3D size distribution determination.

The schematic signification of each class is represented in figure S2. The detail of these classes is given in the UML diagram given in figure S3, The call tree of the main functions is given in figure 54 .

\subsubsection{Optimizations and performances}

A first version of the program only used a Layer class, as a direct two dimensional set of events. A profiling of this program has shown that the process was most of the time being calculating which event was to be performed. As the number of event is proportional to the square of the length of the basis (smax), the time of calculation was roughly proportional to $\mathrm{smax}^{2}$, i.e. it very quickly increased with the size of the simulated crystal. Practically, very long time of calculation were necessary for sufficiently large systems.

An intermediate class Sublayer was thus added between Layer and Event. It allows to stock in memory the intermediate total of stochastic rates of each sublayer. The localization of the appropriate reaction is then only proportional to $2 * \operatorname{smax}$. The profiling of this version shows that their are no more deadlocks, and that the process activity is distributed in the several calculation sections (see figure S4).

A typical simulation can be performed - on a Pentium M processor, $1.8 \mathrm{GHz}, 1 \mathrm{~GB}$ DDRAM - from few minutes (in the cases of a $200 \times 200 \times 40$ crystal), to few hours (in the case of a $300 \times 300 \times 8192$ crystal). This corresponds to about 100,000 to 150,000 elementary reaction per second, an elementary reaction being computed in 7 to $8 \mu \mathrm{s}$.

\subsubsection{Reports}

The python batch program allows the automation of the calculation of several crystallization systems for different parameters. At the end of each simulation,

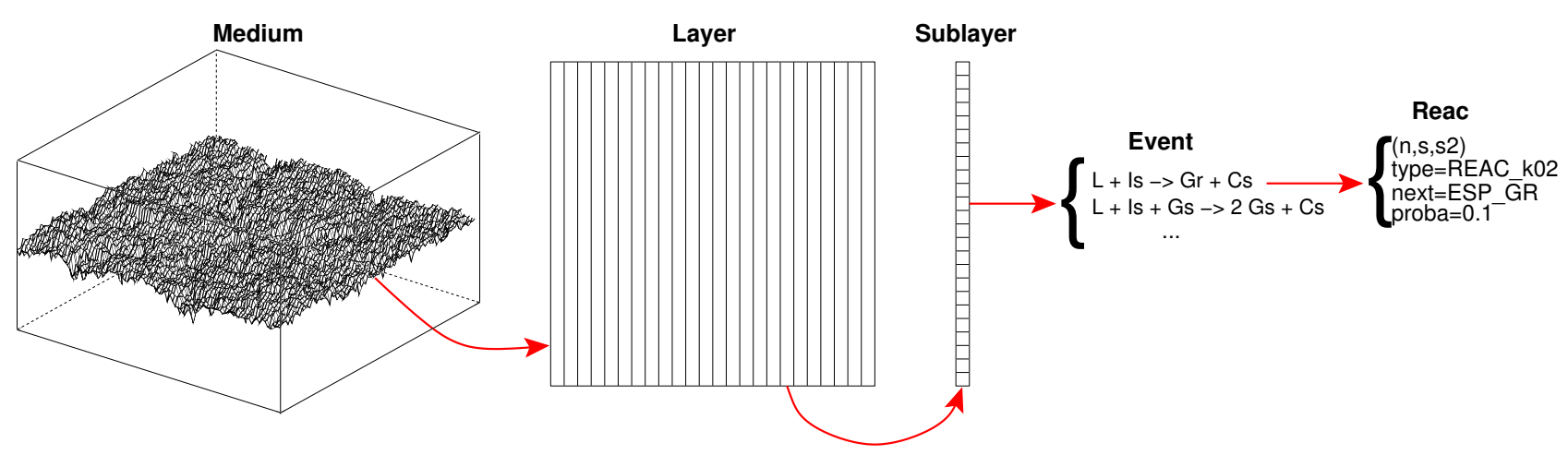

Figure S2: Schematic signification of the program classes. 


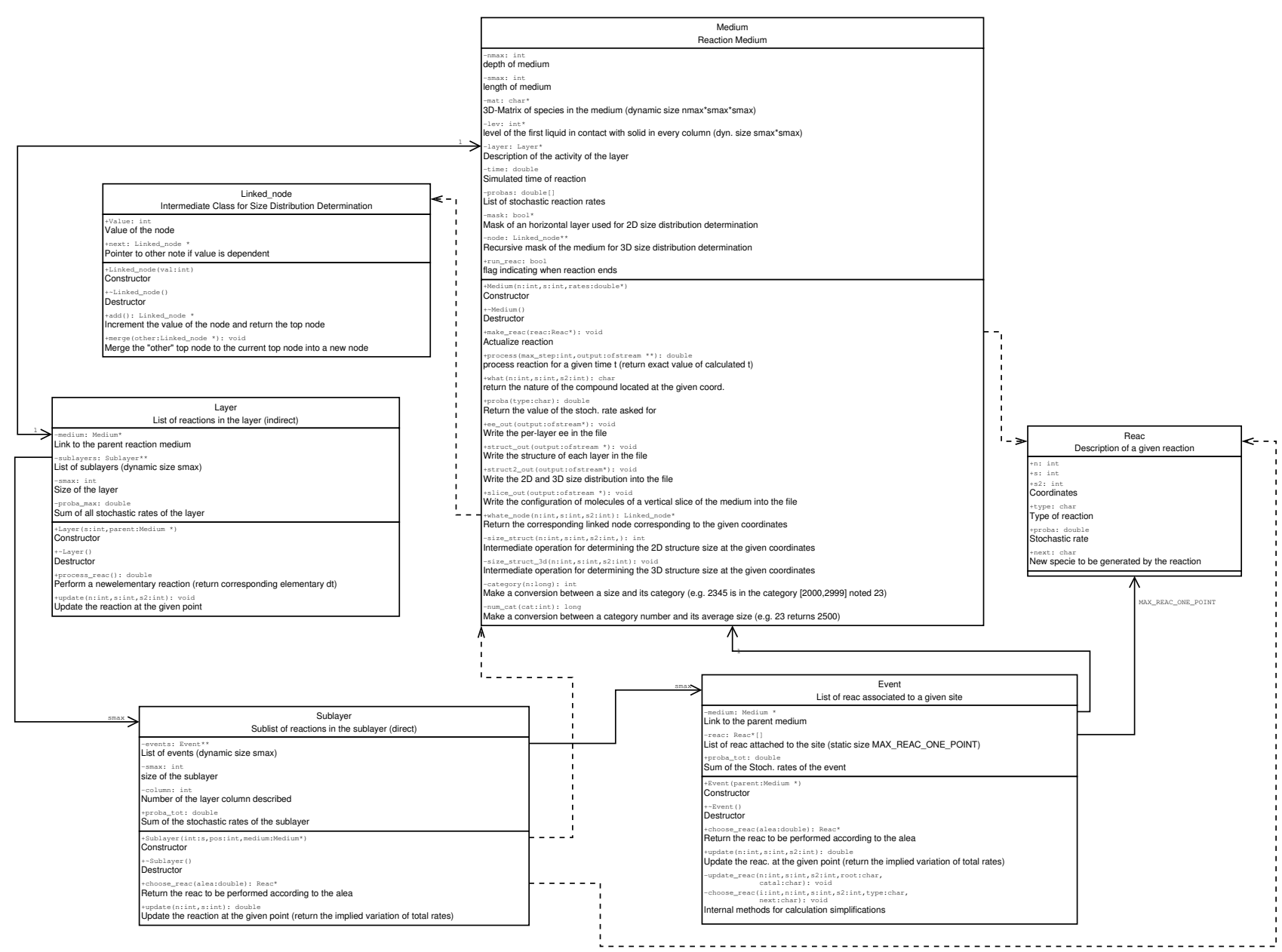

Figure S3: UML Diagram
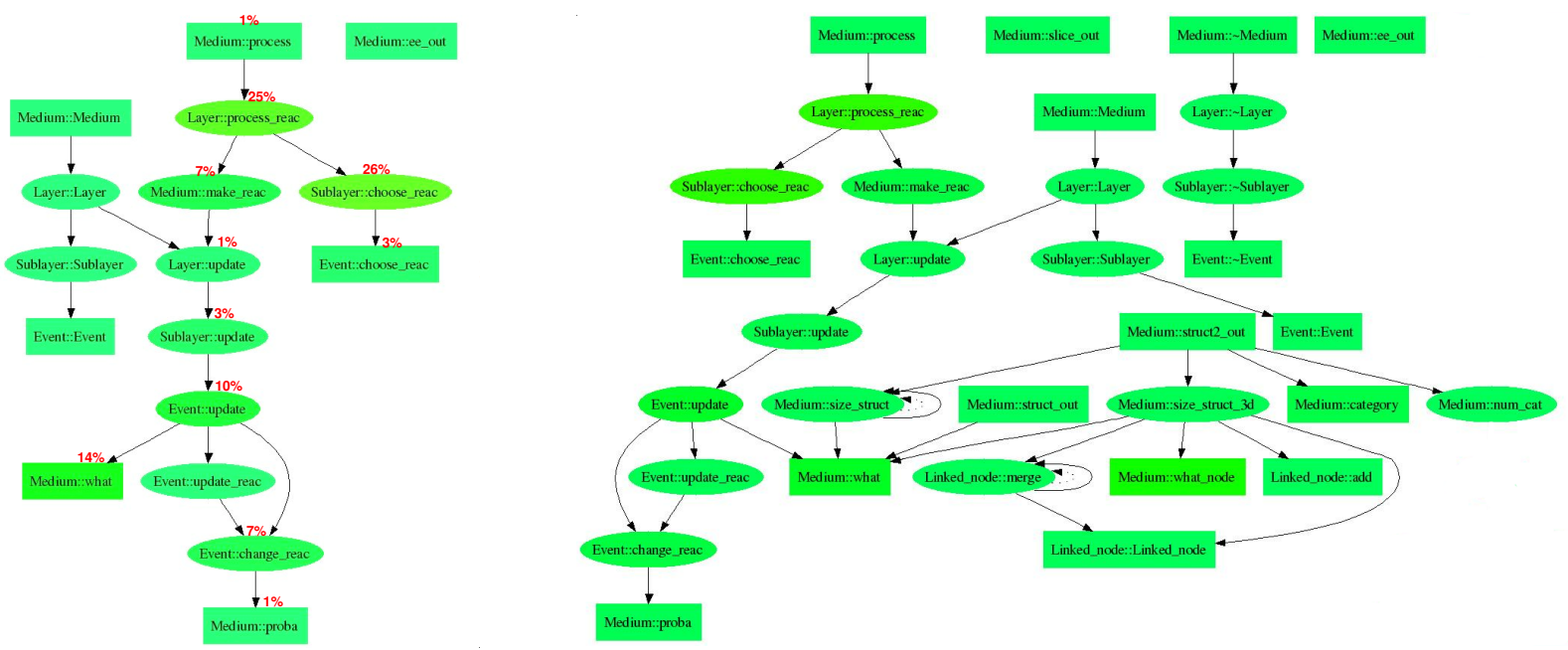

Figure S4: Call tree traced by the GNU profiling tool oprofile. In red are indicated the fraction of time spent by the process in each indicated method (only percentage superior to $1 \%$ and most used methods are given). The first graph is for a run without structure determination, and the second one includes it. 
a one page report is printed. An example of report is given in figure S5. They are all composed of these following parts (from left to right and top to bottom):

- the heading gives the reference of the run (written in blue) and all the parameters of the simulation (written in black).

- up to eight graphic representation of the growth front. Red: $G_{R}$, green: $G_{S}$, light red: $I_{R}$, light green: $I_{S}$. They are given in a chronological order, and correspond to a scan of the growth front at regular time interval.

- A three-dimensional representation of the surface of the growth front. This surface is drawn for the same time than the second flat representation of the growth front.

- The evolution of the level of the growth front as a function of simulation time. The red line indicates the lower altitude of the growth front (in number of molecular layers), and the green line the higher altitude of the growth front.

- The enantiomeric excess of each layer of the final crystal.

- The average size of the homochiral structures of each layer.

\section{Statistical Fluctuations of Enan- tiomeric Excess}

A system having a finite number $n$ of chiral molecules is subject to statistical fluctuations of its enantiomeric excess ee. The evaluation of the value of these fluctuations is a simple combinatorial problem.

\subsection{Calculation}

Given a system of $n$ molecules, each being of either L or D configuration, the number of possibilities for having $p \in[0, n] L$ and $(n-p)$ D equals:

$$
C_{n}^{p}=\frac{n !}{(n-p) ! p !}
$$

The enantiomeric excess of this state is:

$$
e e=\frac{(n-p)-p}{n}
$$

The average enantiomeric excess for all the possible states of the system is zero (by symmetry). The stan- dard deviation around zero is then:

$$
\sqrt{\overline{e e^{2}}}=\sqrt{\frac{\sum_{p=0}^{n} C_{n}^{p}\left(\frac{n-2 p}{n}\right)^{2}}{\sum_{p=0}^{n} C_{n}^{p}}}
$$

This equation can be calculated using this relation:

$$
\sum_{p=0}^{n} C_{n}^{p}=2^{n}
$$

The fluctuations around the racemic state for a system constituted of $n$ molecules is thus:

$$
\sqrt{\overline{e e^{2}}}=\frac{1}{\sqrt{n}}
$$

(see Fig. S6 for details)

\subsection{Numerical application}

The relation 5 implies that for a macroscopic system, fluctuations around the racemic state are low, but may not be negligible. For the simulations that were performed, a common value of $n$ was 65,536 (per layer, that is a square of $256 \times 256$ elementary subunits). Enantiomeric excesses in these simulations are therefore significant when somewhat greater than $4 \cdot 10^{-3}$. It should be noted that the observed fluctuations of enantiomeric excesses are well conforming to this value.

For one mole, $n=\mathcal{N}=6.02 \cdot 10^{23} \Longrightarrow \sqrt{\overline{e e^{2}}} \approx$ $1.3 \cdot 10^{-12}$. This value should be compared to the value given for the estimation of the enantiomeric excess induced by $\operatorname{PVED}^{8}$, i.e. $\varepsilon \in\left[10^{-17}, 10^{-14}\right]$, that is a value 100 times to 100,000 times lower. If we want the effect of the parity violation on the enantiomeric excess not to be obstructed by the statistical fluctuations, we would need between $1.7 \cdot 10^{4}$ and $1.7 \cdot 10^{10}$ moles of compounds, that is in the case of alanine between $1.5 \cdot 10^{3}$ and $1.5 \cdot 10^{9} \mathrm{~kg}$.

\section{Relationship between stochas- tic and macroscopic rates}

\subsection{First order reactions}

\subsubsection{Macroscopic description}

For a given reaction $A \rightarrow B$, in a system containing a molecules of $A$ over a total number $n$ of molecules,

\footnotetext{
${ }^{8}$ Martín Avalos, Reyes Babiano, Pedro Cintas, José L. Jiménez, and Juan C. Palacios. From parity to chirality: chemical implications revisited Tetrahedron: Asymmetry, 11:2845-2874, 2000.
} 


$\begin{array}{lllll}\text { cryst-scan11_0007 tps }=100000000 & p 02=0 & p 12 b=1.0 & \text { eeinit }=0 \\ n=8192 & d s t e p=23040000 & p 11=10.0 & p 13=0 \\ s=300 & \text { p01=10 } & \text { p12a }=1.0 & p 20=10 & \text { s1swp=0 } \\ \text { s } & & & \end{array}$
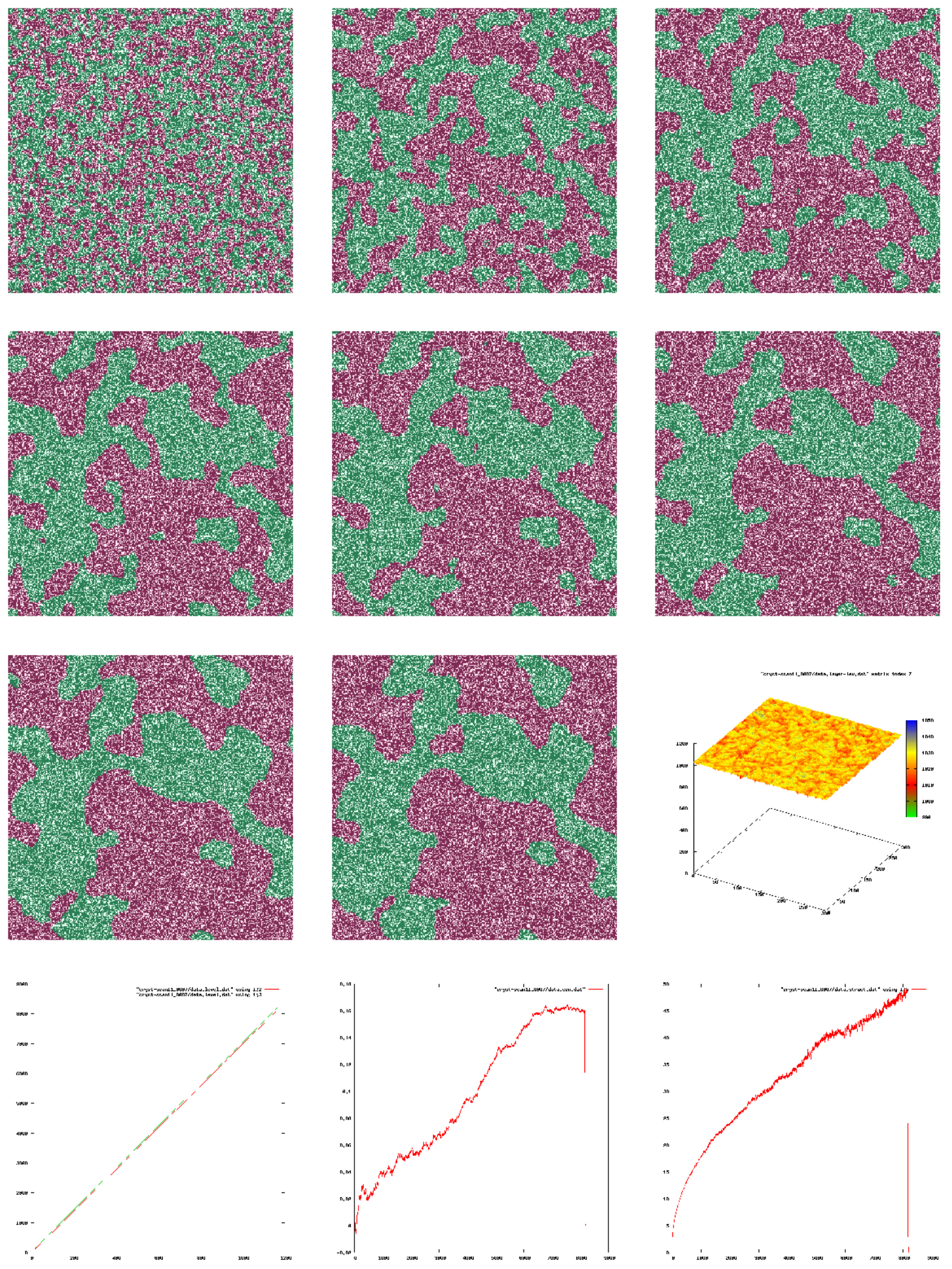

Figure S5: Example of an automatically generated report 


$$
\begin{aligned}
& \sqrt{e e^{2}}=\sqrt{\frac{\sum_{p=0}^{n} C_{n}^{p}\left(\frac{n-2 p}{n}\right)^{2}}{\sum_{p=0}^{n} C_{n}^{p}}} \\
& =\sqrt{2^{-n} \sum_{p=0}^{n} C_{n}^{p} \frac{n^{2}+4 p^{2}-4 n p}{n^{2}}} \\
& =\sqrt{2^{-n}\left(2^{n}+4 \sum_{p=0}^{n} \frac{n ! \cdot p^{2}}{(n-p) ! p ! \cdot n^{2}}-4 \sum_{p=0}^{n} \frac{n ! \cdot p}{(n-p) ! p ! \cdot n}\right)} \\
& =\sqrt{1+2^{2-n}\left(\sum_{p=1}^{n} \frac{(n-1) ! \cdot p}{(n-p) !(p-1) ! \cdot n}-\sum_{p=1}^{n} \frac{(n-1) !}{(n-p) !(p-1) !}\right)} \\
& =\sqrt{1+2^{2-n}\left(\sum_{p=0}^{n-1} \frac{(n-1) !}{(n-p-1) ! p !} \cdot \frac{p+1}{n}-\sum_{p=0}^{n-1} \frac{(n-1) !}{(n-p-1) ! p !}\right)} \\
& =\sqrt{1+2^{2-n}\left(\sum_{p=0}^{n-1} \frac{p}{n} \cdot C_{n-1}^{p}+\frac{1}{n} \sum_{p=0}^{n-1} C_{n-1}^{p}-\sum_{p=0}^{n-1} C_{n-1}^{p}\right)} \\
& =\sqrt{1+2^{2-n}\left(\sum_{p=1}^{n-1} \frac{n-1}{n} \cdot \frac{(n-2) !}{(n-1-p) !(p-1) !}+\frac{2^{n-1}}{n}-2^{n-1}\right)} \\
& =\sqrt{1+2^{2-n} \cdot \frac{n-1}{n} \cdot \sum_{p=0}^{n-2} C_{n-2}^{p}+\frac{2}{n}-2} \\
& =\sqrt{\frac{n-1}{n}+\frac{2}{n}-1} \\
& =\frac{1}{\sqrt{n}}
\end{aligned}
$$

Figure S6: Calculation of the standard deviation of the enantiomeric excess around the racemic state in a system of $n$ molecules. 
we have the following parameters:

$$
\begin{aligned}
& x_{a}=\frac{a}{n} \\
& v_{a}=\frac{d x_{a}}{d t} \\
& v_{a}=k_{a} x_{a}
\end{aligned}
$$

where $x_{a}$ is the molar fraction of $A, v_{a}$ the rate of variation of $A$, and $k_{a}$ the kinetic (macroscopic) rate of $A$ consumption (in $\mathrm{s}^{-1}$ ).

\subsubsection{Stochastic description}

In the same system, at a given time, one given molecule of $A$ will react after an average time $\delta t=$ $\frac{1}{p_{a}}, p_{a}$ being the stochastic rate of A consumption (in $\mathrm{s}^{-1}$ ). For the subsystem constituted by this unique molecule, we have $\delta a=1$ during this elementary $\delta t$.

For a system constituted by a molecules of $A$, the total probability of all elementary steps is $a p_{a}$. One reaction - corresponding to $\delta a=1-$ will occur in this system after the elementary time $\delta t=\frac{1}{a p_{a}}$. We thus obtain:

$$
\begin{aligned}
\frac{\delta a}{\delta t} & =a p_{a} \\
\Rightarrow \frac{\delta x_{a}}{\delta t} & =x_{a} p_{a}
\end{aligned}
$$

At the limit of large values of $A$, we thus have:

$$
\mathrm{p}_{\mathrm{a}}=\mathrm{k}_{\mathrm{a}}
$$

This corresponds to the case $G_{x} \rightarrow I_{x}$ in the studied simulations.

\subsection{Reaction in a surface site}

For a given reaction $A+I \rightarrow B$ ( $A$ corresponding to a liquid compound that can react on sites I of a surface) the case is formally identical to the preceding one. As all I sites are surrounded by a $A$ compound (all liquid compounds being identical), this reaction is formally identical to a first order reaction of one $[A+I]$ compound, of molar fraction $x_{i}$. Here again, macroscopic and stochastic rates are equal. This corresponds to the cases $\mathrm{L}+\mathrm{I}_{x} \rightarrow \mathrm{G}_{x}+\mathrm{C}_{x}$.

\subsection{Second order reaction in a surface site}

\subsubsection{Macroscopic description}

For a given reaction $A+I+C \rightarrow B$ ( $A$ being a liquid phase surrounding all sites) corresponding to a reaction on a site $\mathrm{I}$, catalyzed by a neighboring site $\mathrm{C}$, we have the following parameters:

$$
\begin{aligned}
s & =\frac{\mathcal{N s}}{n} \\
x_{i} & =\frac{i}{n} \\
x_{c} & =\frac{c}{n} \\
\sigma_{i} & =\frac{x_{i}}{S} \\
\sigma_{c} & =\frac{x_{c}}{S} \\
v_{i} & =\frac{d \sigma_{i}}{d t} \\
& =k_{i c} \sigma_{i} \sigma_{c}
\end{aligned}
$$

where $S$ is the molar surface of the crystal (i.e. the surface occupied by one mole of sites), $\mathcal{N}$ the Avogadro number, $s$ the total surface of the crystal, $n$ the number of sites on the surface $s, \sigma_{i}$ and $\sigma_{c}$ the surfacic concentration of respectively $I$ and $C, i$ and $c$ the number of sites I and $C$ on the surface $s, x_{i}$ and $x_{c}$ the fraction of sites occupied by I and C, $v_{i}$ the rate of variation of $I$, and $k_{i c}$ the kinetic (macroscopic) rate of I consumption (in $\mathrm{mol}^{-1} \cdot \mathrm{m}^{2} \cdot \mathrm{s}^{-1}$ ). This kinetic rates expression makes implicitly the assumption that there is an homogeneous distribution of sites on the crystal surface.

\subsubsection{Stochastic description}

For a given subsystem constituted by one site on the surface of the total system, the probability of reaction is:

$p= \begin{cases}0 & \text { site not occupied by I } \\ 0 & \text { site occupied by I, no neighboring C } \\ n p_{i c} & \text { site occupied by I, with neighboring C }\end{cases}$

The probability that one given neighboring site of the subsystem is occupied by $C$ is $\frac{c}{n}$. Thus, assuming that the sites are uniformly distributed on the surface, the total probability of the subsystem to react is:

$$
p=4 \frac{c}{n} p_{i c}
$$

One should notice that this expression is actually an approximation. Actually, if a first neighboring site is occupied by $\mathrm{C}$, the probability that a second neighboring site is occupied by $C$ will be $\frac{c-1}{n}$. The total probability should thus become:

$$
p=\frac{4 c-6}{n} p_{i c}
$$

However, the correction term is proportional to $\mathrm{n}^{-1}$, and will become negligible for a macroscopic system. Thus, only the equation 20 will be considered. 
For the whole system, the total probability of all elementary steps is then:

$$
\begin{aligned}
p & =4 \frac{i c}{n} p_{i c} \\
& =\frac{\delta i}{\delta t} \\
\Rightarrow \frac{\delta \sigma_{i}}{\delta t} & =\frac{1}{n s} \frac{\delta i}{\delta t} \\
& =4 S \sigma_{i} \sigma_{c} p_{i c}
\end{aligned}
$$

At the limit of large dimension of an homogeneous system, we thus have:

$$
k_{i c}=4 S p_{i c}
$$

$k_{i c}$ is in $\mathrm{mol}^{-1} \cdot \mathrm{m}^{2} \cdot \mathrm{s}^{-1}, \mathrm{~S}$ in $\mathrm{mol}^{-1} \cdot \mathrm{m}^{2}$ and $p_{i c}$ in $s^{-1}$. This corresponds to the case $L+I_{x}+G_{y} \rightarrow$ $\mathrm{G}_{z}+\mathrm{C}_{\mathrm{x}}+\mathrm{G}_{\mathrm{y}}$ in the studied simulations.

\section{Program Listing}

\subsection{ODE file of the homogeneous model}

This is the .ode file used for the numeric integration of the homogeneous model of frontal crystallization by the XppAut software.

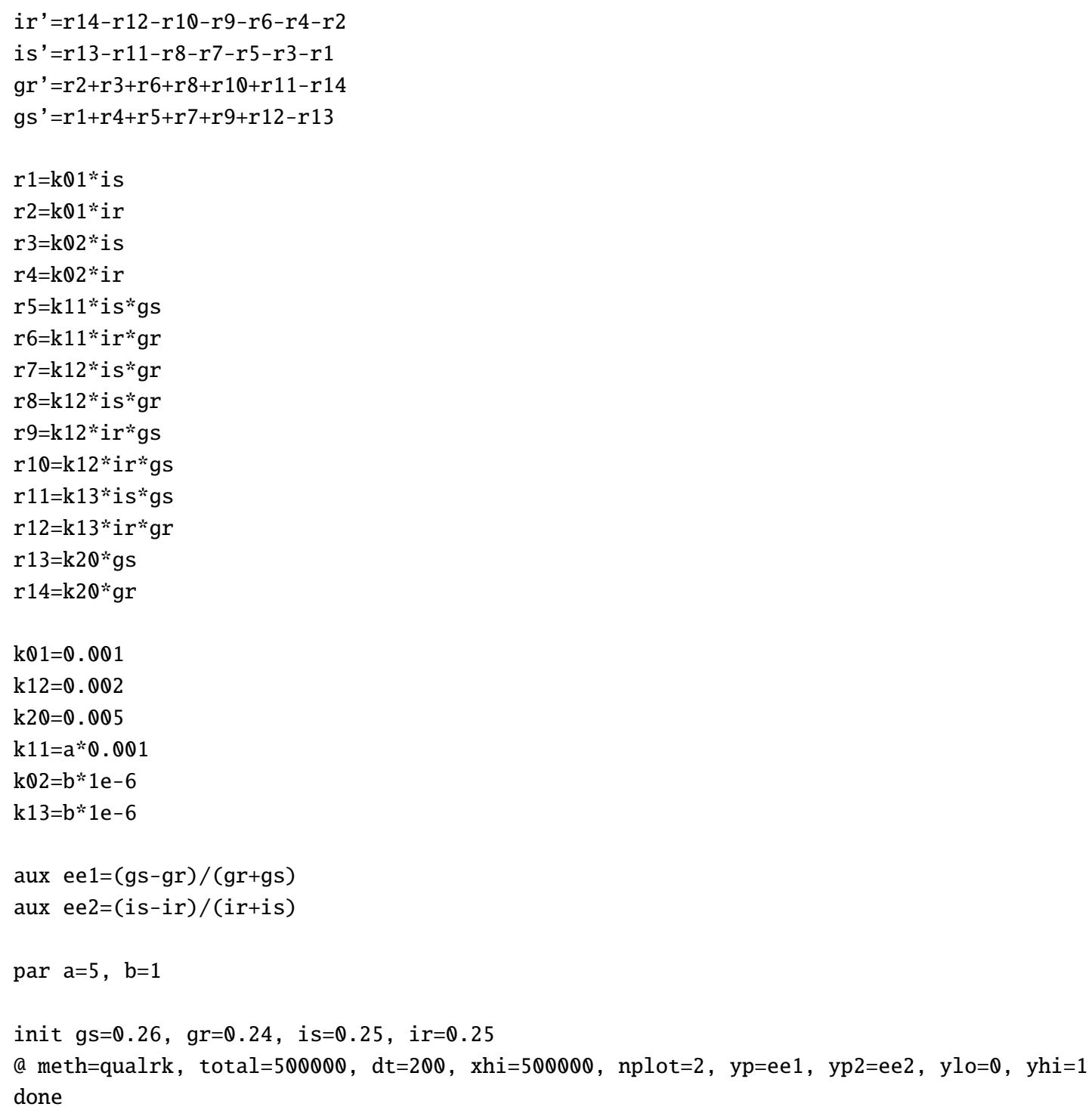

\subsection{Main Program}

\subsection{1 include.h}

There are general definitions and includes. 


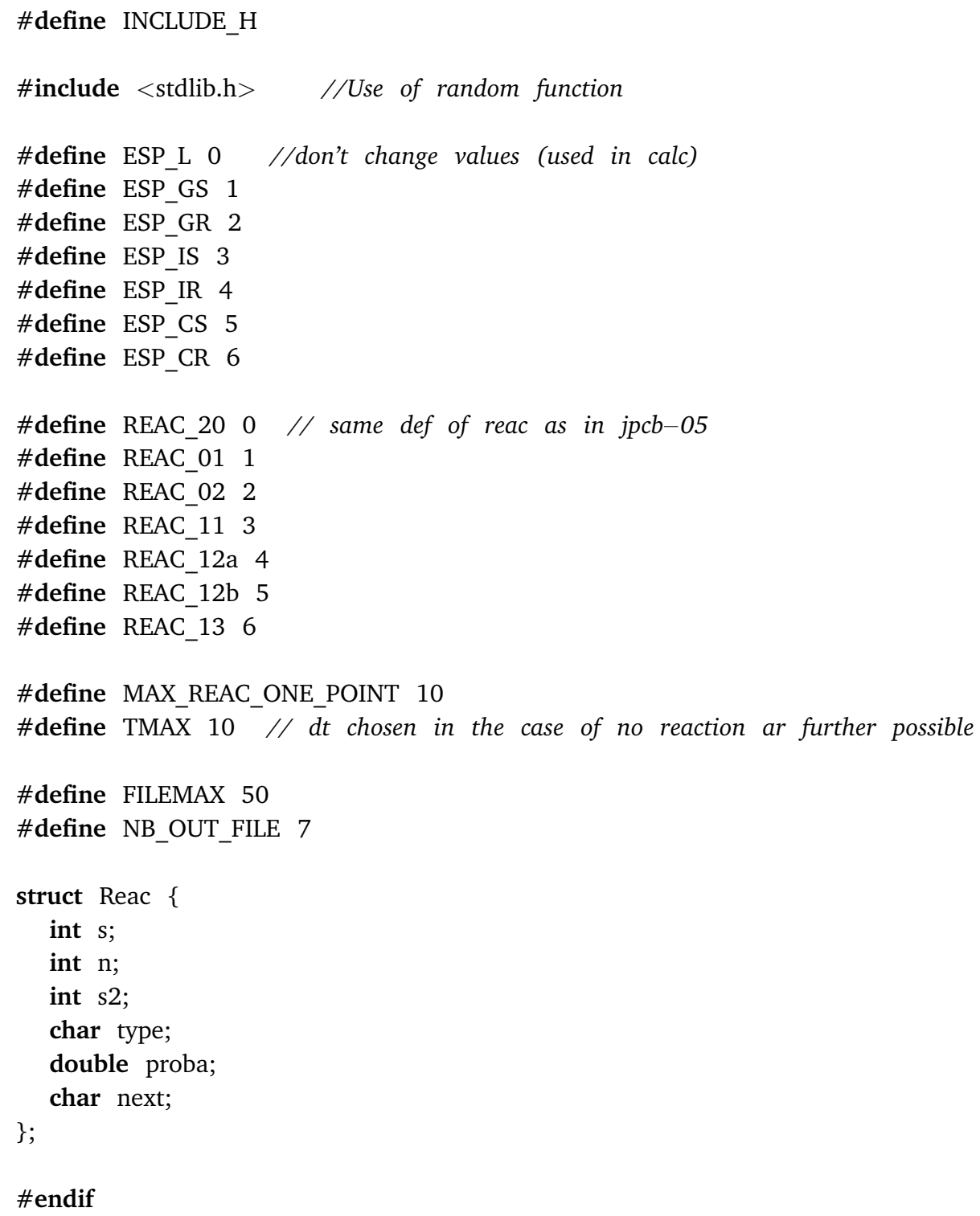

\subsection{2 cryst.cpp}

This is the main program, that initiates calculations, read the parameters file and generates the output files.

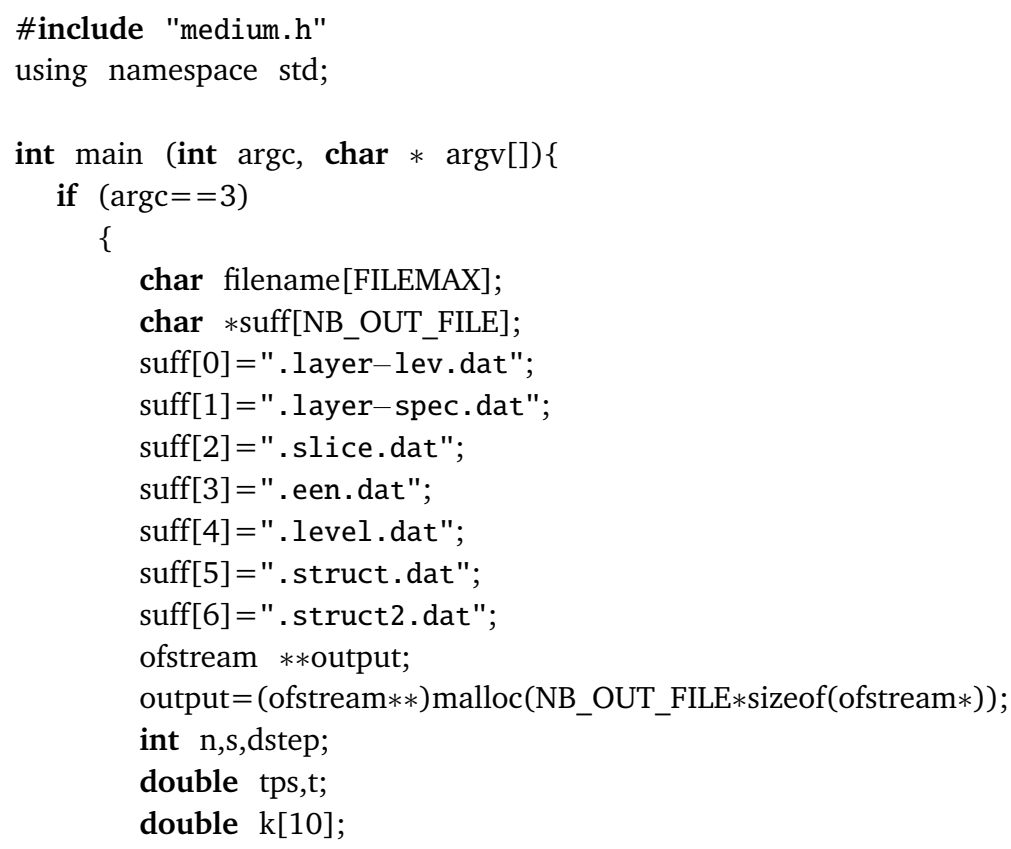


Medium $*$ medium;

//seed from urandom

char data[4];

long seed;

ifstream random ("/dev/urandom", ios::in);

random.read(data, $4 * \operatorname{sizeof}($ char));

seed $=$ data $[0]+128+($ data $[1]+128) * 256+($ data $[2]+128) * 256 * 256+($ data $[3]+128) * 256 * 256 * 256$;

srand48(seed);

random.close();

ifstream param(argv[1], ios::in);

for (int $i=0 ; i<$ NB_OUT_FILE; $i++$ )

\{

strcpy(filename, $\operatorname{argv}[2])$;

strcat(filename,suff[i]);

$*($ output $+\mathrm{i})=$ new ofstream(filename, ios::out);

\}

//read parameters

param $>>\mathrm{n}$;

cout $<<$ "n=" $<<\mathrm{n} \quad<<$ "\n";

param $>>s$;

cout $<<$ "s=" $<<\mathrm{s} \quad<<$ " $\backslash \mathrm{n}$;

param $>>$ tps;

cout $<<$ "tps=" $<<$ tps $<<$ " \n";

param $>>$ dstep;

cout $<<$ "dt=" $<<$ dstep $<<$ "\n";

param $>>$ k[REAC_01];

cout $<<$ "k01=" $<<$ k[REAC_01] $<<$ "\n";

param $>>$ k[REAC_02];

cout $<<$ "k02=" $<<$ k[REAC_02] $<<$ " \n";

param $>>$ k[REAC_11];

cout $<<$ "k11=" $<<$ k[REAC_11] $<<$ "\n";

param $>>$ k[REAC 12a];

cout $<<$ "k12a=" $<<$ k[REAC_12a] $<<$ " \n";

param $>>$ k[REAC_12b];

cout $<<$ "k12b=" $<<$ k[REAC_12b] $<<$ "\n";

param $>>$ k[REAC_13];

cout $<<$ "k13=" $<<$ k[REAC_13] $<<$ "\n";

param $>>$ k[REAC_20];

cout $<<$ "k20=" $<<$ k[REAC_20] $<<$ "\n";

param $>>\mathrm{k}[7]$;

cout $<<$ "ee_init=" $<<\mathrm{k}[7] \quad<<$ "\n";

param $>\mathrm{k}[8]$;

cout $<<$ "s1swp=" $<<\mathrm{k}[8]<<$ "\n";

param $>\mathrm{k}[9]$;

cout $<<$ "s2swp=" $<<\mathrm{k}[9] \quad<<" \backslash \mathrm{n}$;

// Initialize simulator

medium=new Medium(n,s,k);

// Process

for $(\mathrm{t}=0 ; \mathrm{t}<\mathrm{tps} \& \&$ medium $->$ run_reac; $\mathrm{t}+=$ medium $->$ process(dstep,output));

cout $<<$ "Run $\mathrm{OK} \backslash \mathrm{n}$ ";

medium->ee_out $(*($ output +3$))$;

medium- $->$ slice_out $(*($ output +2$))$;

medium- $>$ struct_out $(*$ (output +5$))$;

medium->struct2_out $(*($ output +6$))$;

for (int $\mathrm{i}=0 ; \mathrm{i}<\mathrm{NB} \_$OUT_FILE; $\left.\mathrm{i}++\right)(*($ output $+\mathrm{i}))->\operatorname{close}()$;

free(output);

delete medium;

cout $<<$ "End of Program :-) \n";

return 0 ; 


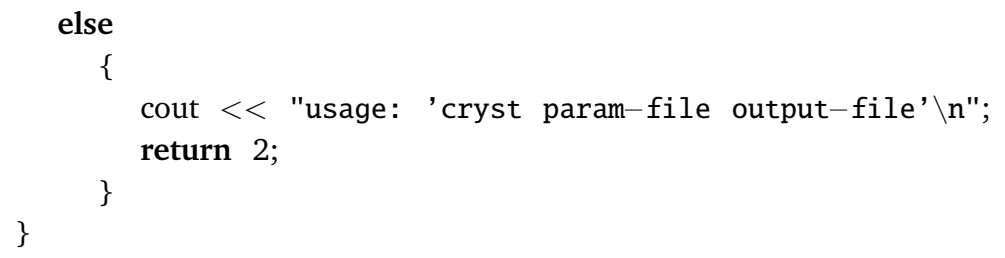

\subsubsection{Makefile}

Makefile used for the generation of the executable file program.

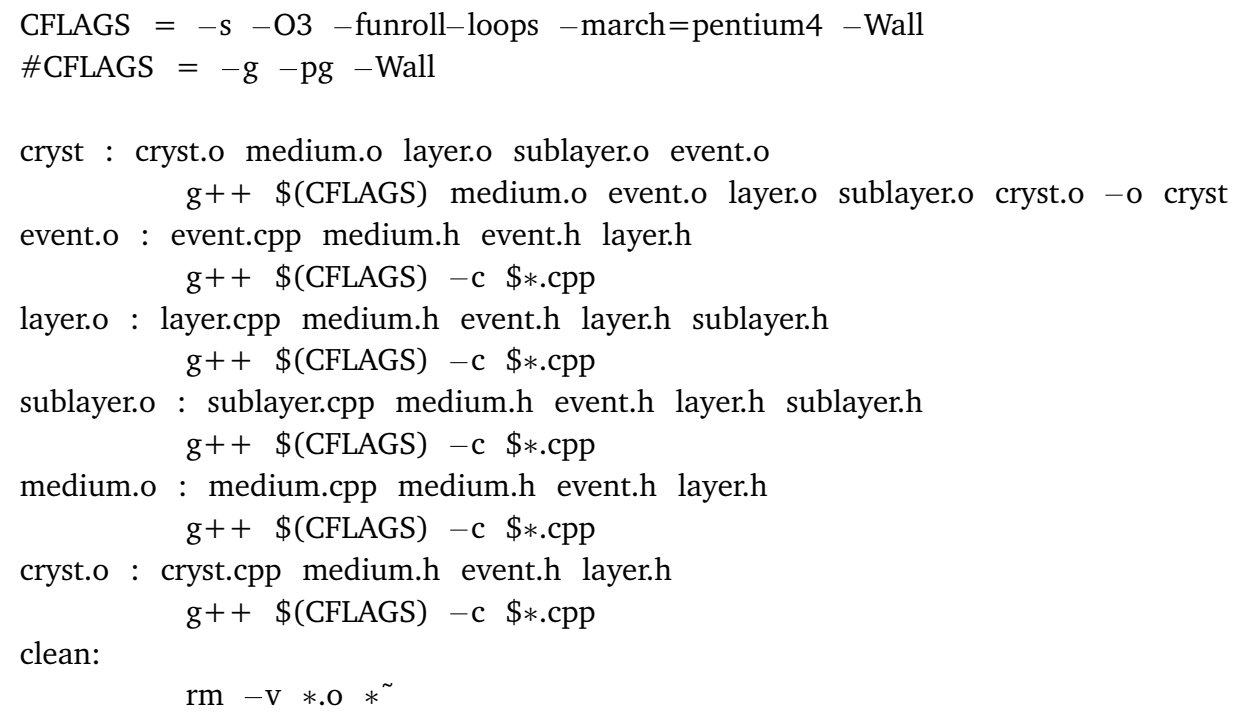

\subsection{4 batch.py}

This is the batch python program, used for calling the calculation program for several set of parameters, generating and printing a report page for each performed calculation, and compressing all the output files in a single .tar.bz2 archive file.

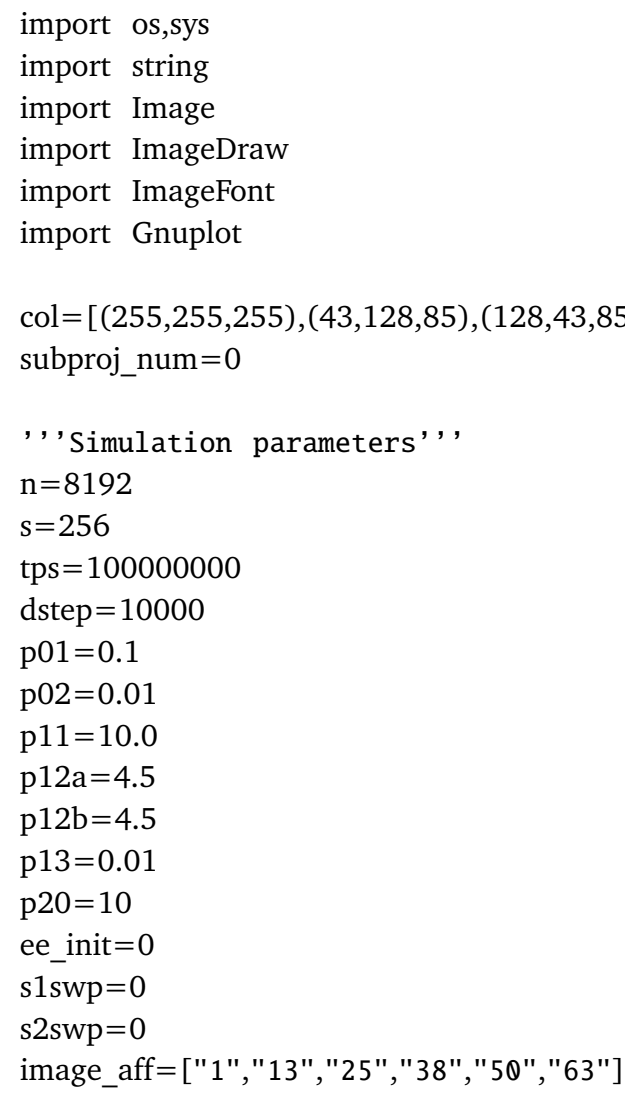


layer_lev="7"

project_name $=$ "cryst - scan $28 "$

for $\mathrm{p} 12 \mathrm{a}$ in $(3.6$,$) :$

$\mathrm{p} 12 \mathrm{~b}=\mathrm{p} 12 \mathrm{a}$

for ee_init in $(0$,$) :$

for rep in range(2):

dstep $=\mathrm{s} * \mathrm{~s} * \mathrm{n} / 32$

, 'Create param file,,

subproj_num $=$ subproj_num +1

subproj_name $=" 00000000 "+\operatorname{str}($ subproj_num)

subproj_name=project_name+"_"+subproj_name[-4:]

os.mkdir(subproj_name)

$\operatorname{print}(" \backslash n \backslash n$ $\left.\backslash \mathrm{n}^{\prime \prime}\right)$

os.system("date")

print("Subproject "+subproj_name+" started. $\backslash \mathrm{n} ")$

param_file=open(subproj_name+"/param","w")

param_file.write(str(n)+"\n")

param_file.write(str(s)+"\n")

param_file.write(str(tps) + "\n")

param_file.write(str(dstep)+" $\backslash \mathrm{n}$ ")

param_file.write(str(p01)+" $\backslash \mathrm{n}$ ")

param_file.write(str(p02)+" $\backslash \mathrm{n}$ ")

param_file.write(str(p11)+" $\backslash \mathrm{n}$ ")

param_file.write(str(p12a)+" $\backslash \mathrm{n} ")$

param_file.write(str(p12b)+" $\backslash \mathrm{n} ")$

param file.write(str(p13)+"\n")

param_file.write(str(p20)+" $\backslash \mathrm{n}$ ")

param_file.write(str(ee_init) +" $\backslash n$ ")

param_file.write(str(s1swp)+" $\backslash n ")$

param_file.write(str(s2swp) + " $\backslash n ")$

param_file.write('\nFile automatically generated. \nProject "'+subproj_name+'". \n')

param file.close()

", 'Calculation, , ,

os.system("unbuffer ./cryst "+subproj_name+"/param "+subproj_name+"/data ")

",'Process images,",

infile=subproj name+"/data. layer-spec.dat"

fin=open(infile, "r")

image =Image.new ("RGB",(s,s))

$\mathrm{k}=0$

rdl=fin.readline()

try:

while $\mathrm{rdl} !=$, ,

$\mathrm{k}=\mathrm{k}+1$

kstr $=" 0000000000 "+\operatorname{str}(\mathrm{k})$

try:

for $\mathrm{i}$ in range(s):

ligne $=$ fin. readline ()

liste $=$ string.split(ligne $)$

for $\mathrm{j}$ in range(s):

color $=$ int $($ liste $[\mathrm{j}])$

if $($ color $>6$ or color $<0)$ :

color $=7$

image.putpixel((i,s-j-1),col[color])

except:

print('Woops!!!!' )

print('i:')

print(i) 


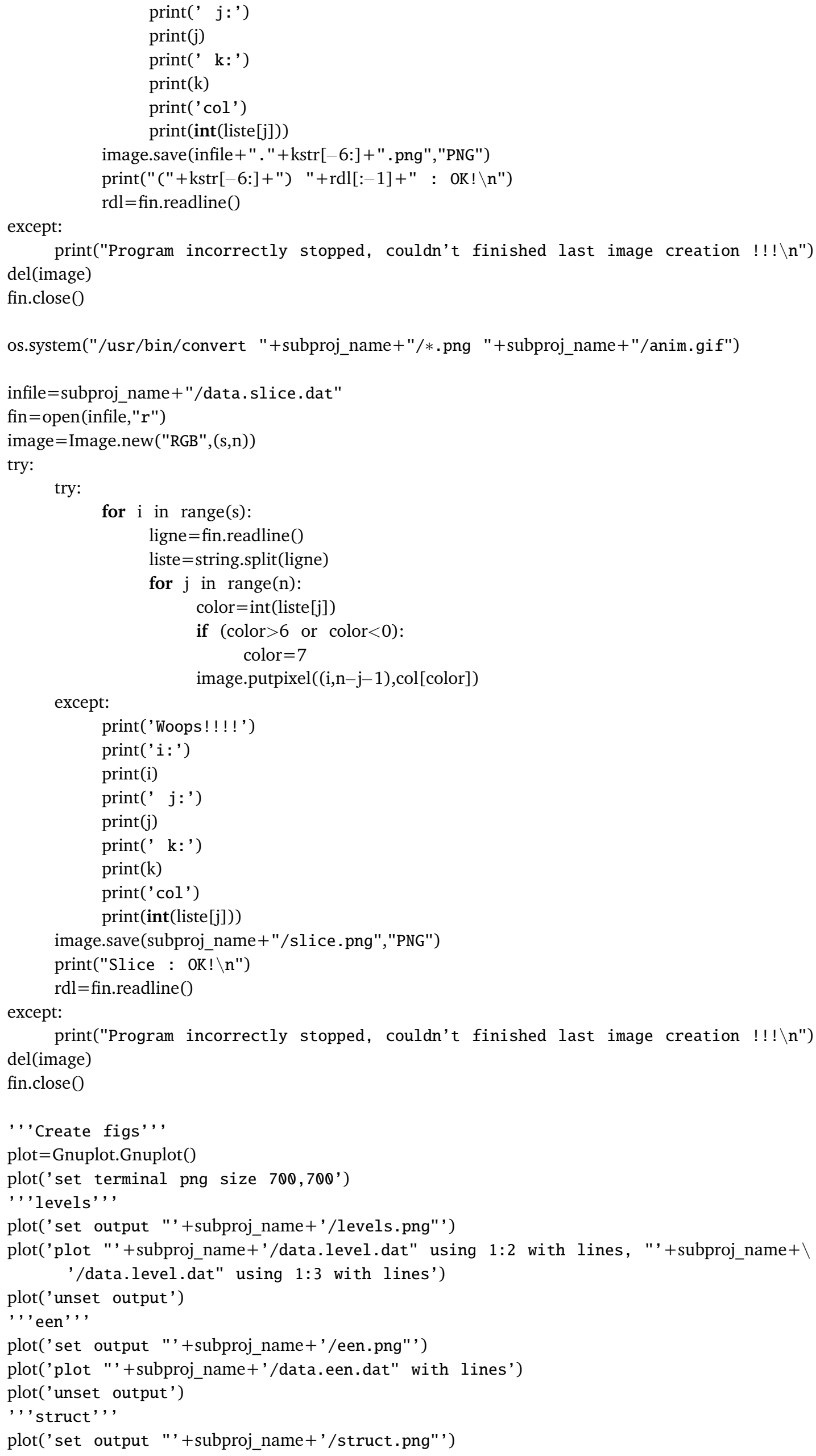


plot('plot "'+subproj_name+'/data.struct.dat" using 1:5 with lines')

plot('unset output')

, , 'surface, ,'

plot('set output "'+subproj_name+'/surface.png"')

plot('set pm3d')

plot('set ticslevel $\theta^{\prime}$ ')

plot('set palette defined ( 0 "green" , 1 "red", 2 "yellow" , 3 "blue")')

plot('splot [0:'+str(s)+'] [0:'+str(s)+'] [0:] "'+subproj_name+

'/data.layer-lev.dat" matrix index '+layer_lev+'with pm3d')

plot('unset output')

, , , struct2, , ,

plot('set output "'+subproj_name+'/struct2.png"')

plot('set logscale')

plot('plot "'+subproj_name+'/data.struct2.dat" index ' $+\operatorname{str}(n-1)+\backslash$

, using $((\operatorname{sgn}(\$ 1)+1) / 2 * \$ 1):(\operatorname{abs}(\$ 2))$ with lines smooth unique, "’

+subproj_name+'/data.struct2.dat" index '+str(n-1)+

, using $((\operatorname{sgn}(\$ 1)-1) / 2 * \$ 1):(\operatorname{abs}(\$ 2))$ with lines smooth unique, "'। +subproj_name+'/data.struct2.dat" index ' + str(n) $+\backslash$

' using $((\operatorname{sgn}(\$ 1)+1) / 2 * \$ 1):(\operatorname{abs}(\$ 2))$ with lines smooth unique, "' $\backslash$

+subproj_name+'/data.struct2.dat" index ' $+\operatorname{str}(\mathrm{n})+\backslash$

' using $((\operatorname{sgn}(\$ 1)-1) / 2 * \$ 1):(\operatorname{abs}(\$ 2))$ with lines smooth unique')

plot('unset output')

plot('exit')

del(plot)

, ,'Create report, , ,

report=Image.new("RGB",(2480,3508),(255,255,255))

, , ' $\mathrm{n}=0$, , ,

kstr $=" 0000000000 "+$ image_aff[0]

try:

im=Image.open(subproj_name+' /data . layer-spec.dat .' +kstr[-6:]+ '.png')

scaled $=$ im.resize $((700,700))$

report.paste(scaled, $(95,328,795,1028))$

except IOError:

print $(k s t r[-6:]+"$ not here $\backslash n ")$

, , 'n=1, , ,

kstr $=$ "0000000000"+image_aff[1]

try:

im=Image.open(subproj_name+'/data.layer-spec.dat.' +kstr[-6:]+ '.png')

scaled $=$ im.resize $((700,700))$

report.paste(scaled, $(890,328,1590,1028)$ )

except IOError:

print $(k s t r[-6:]+"$ not here $\backslash n ")$

, , , $\mathrm{n}=2$, , ,

kstr $=" 0000000000 "+$ image_aff[2]

try:

im=Image.open(subproj_name+' /data . layer-spec.dat .' +kstr[-6:]+ '.png')

scaled $=$ im.resize $((700,700))$

report.paste(scaled, $(1685,328,2385,1028)$ )

except IOError:

print $(k s t r[-6:]+"$ not here $\backslash \mathrm{n} ")$

, , ' $\mathrm{n}=3$, , ,

kstr $=" 0000000000 "+$ image_aff[3]

try:

im =Image.open(subproj_name+'/data . layer-spec.dat .' +kstr[-6:]+ '.png')

scaled $=$ im.resize $((700,700))$

report.paste(scaled, $(95,1123,795,1823))$

except IOError:

$\operatorname{print}(k s t r[-6:]+"$ not here $\backslash \mathrm{n} ")$

, , 'n=4, , ,

kstr $=" 0000000000 "+$ image_aff[4] 
try:

im =Image.open(subproj_name+' '/data.layer-spec. dat. ' +kstr[-6:]+' '. png')

scaled $=$ im.resize $((700,700))$

report.paste(scaled, $(890,1123,1590,1823))$

except IOError:

$\operatorname{print}(k s t r[-6:]+"$ not here $\backslash \mathrm{n} ")$

, , 'n=5, , ,

kstr $=" 0000000000 "+$ image_aff[5]

try:

im =Image.open(subproj_name+' '/data. layer-spec. dat. ' +kstr[-6:]+' '.png')

scaled $=$ im.resize $((700,700))$

report.paste(scaled, $(1685,1123,2385,1823))$

except IOError:

$\operatorname{print}(\mathrm{kstr}[-6:]+"$ not here $\backslash \mathrm{n} ")$

, , slice, ,

try:

im =Image.open(subproj_name+' '/slice.png')

scaled $=$ im.resize $((700,700))$

report.paste(scaled, $(95,1918,795,2618))$

except IOError:

print("Slice not here $\backslash \mathrm{n} ")$

, , 'struct2, , ,

try:

im =Image.open(subproj_name+' '/struct2. png')

report.paste(im, $(890,1918,1590,2618))$

except IOError:

print("Struct2 not here $\backslash \mathrm{n}$ ")

, , 'report. save("/tmp/1.png", "PNG"), , ,

",'levels",

try:

im =Image.open (subproj_name+' '/levels.png')

report.paste(im, $(95,2713,795,3413))$

except IOError:

print("levels not here $\backslash \mathrm{n} ")$

, , 'een', ,

try:

im =Image.open(subproj_name+ ' /een.png')

report.paste(im, $(890,2713,1590,3413))$

except IOError:

print("een not here $\backslash \mathrm{n} ")$

", 'struct", ,

try:

im =Image.open(subproj_name+ '/struct.png')

report.paste(im, $(1685,2713,2385,3413))$

except IOError:

print("struct not here $\backslash \mathrm{n} ")$

, , surface", ,

try:

im =Image.open(subproj_name +' /surface.png')

report.paste(im, $(1685,1918,2385,2618))$

except IOError:

print("surface not here $\backslash n$ ")

del(im)

del(scaled)

", 'finish report,",

draw=ImageDraw.Draw(report)

fnt=ImageFont.load("helv . pil")

draw.text $((95,95)$,subproj_name, fill $=(0,0,255)$, ,font $=$ fnt $)$

draw.text $((95,141), " n="+\operatorname{str}(n)$, fill $=(0,0,0)$, font $=$ fnt $)$

draw.text $((95,187), " s="+\operatorname{str}(s)$, fill $=(0,0,0)$,font $=$ fnt $)$

draw.text((553,95),"tps=" $+\operatorname{str}($ tps $)$, fill $=(0,0,0)$, font $=$ fnt $)$ 


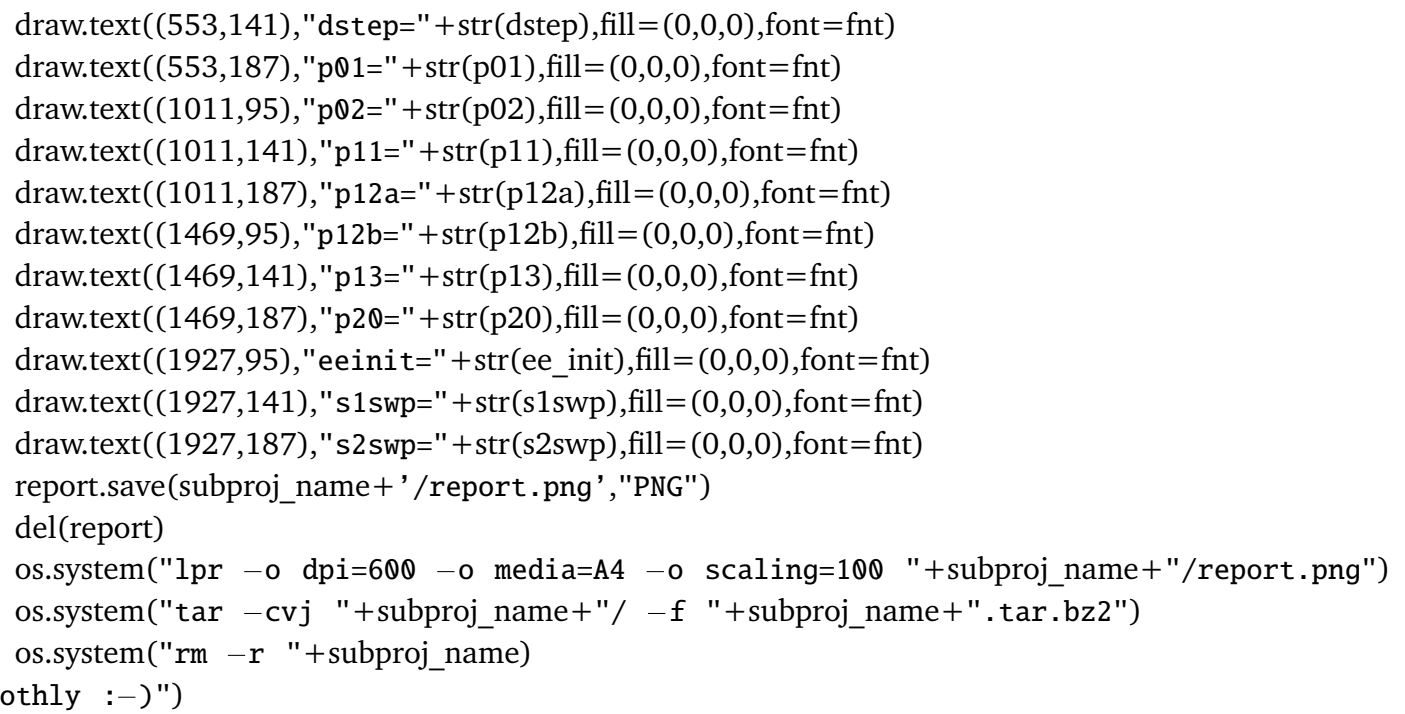

\subsection{Medium Class}

This class contain the 3-D matrix describing the growing crystal, and its link (via layer and sublayer classes) with possible reactions (described in event class).

\subsection{1 medium.h}

\#ifndef MEDIUM_H \#define MEDIUM_H

\#include "include.h"

\#include "layer.h"

\#include <iostream $>$

\#include $<$ fstream $>$

using namespace std;

class Layer;

class Reac;

class Linked node;

class Medium \{

// Attributes

private:

double time;

int nmax;

int smax;

char* mat;

int* lev;

double probas[7];

Layer $*$ layer;

bool $*$ mask;

Linked node $* *$ node;

public:

bool run_reac;

// Operations

public:

Medium(int , int, double*);

Medium();

void make_reac ( Reac * reac);

double process ( int, ofstream $* *$ );

char what(int,int,int);

Linked_node* what_node(int,int,int);

double ${ }^{-}$proba(char); 


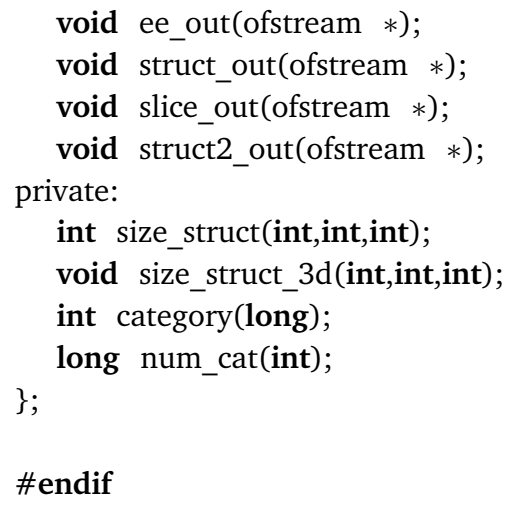

\subsection{2 medium.cpp}

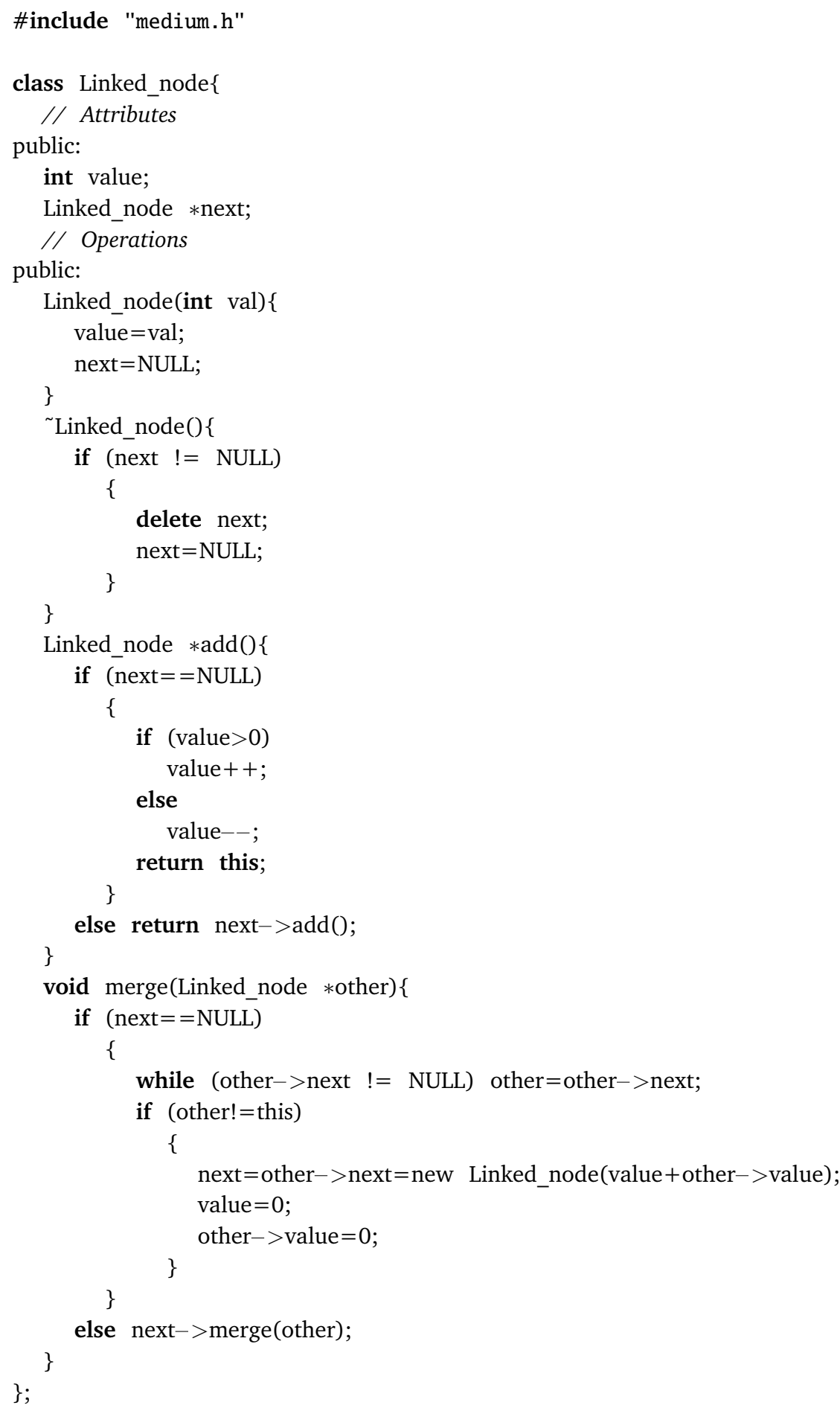




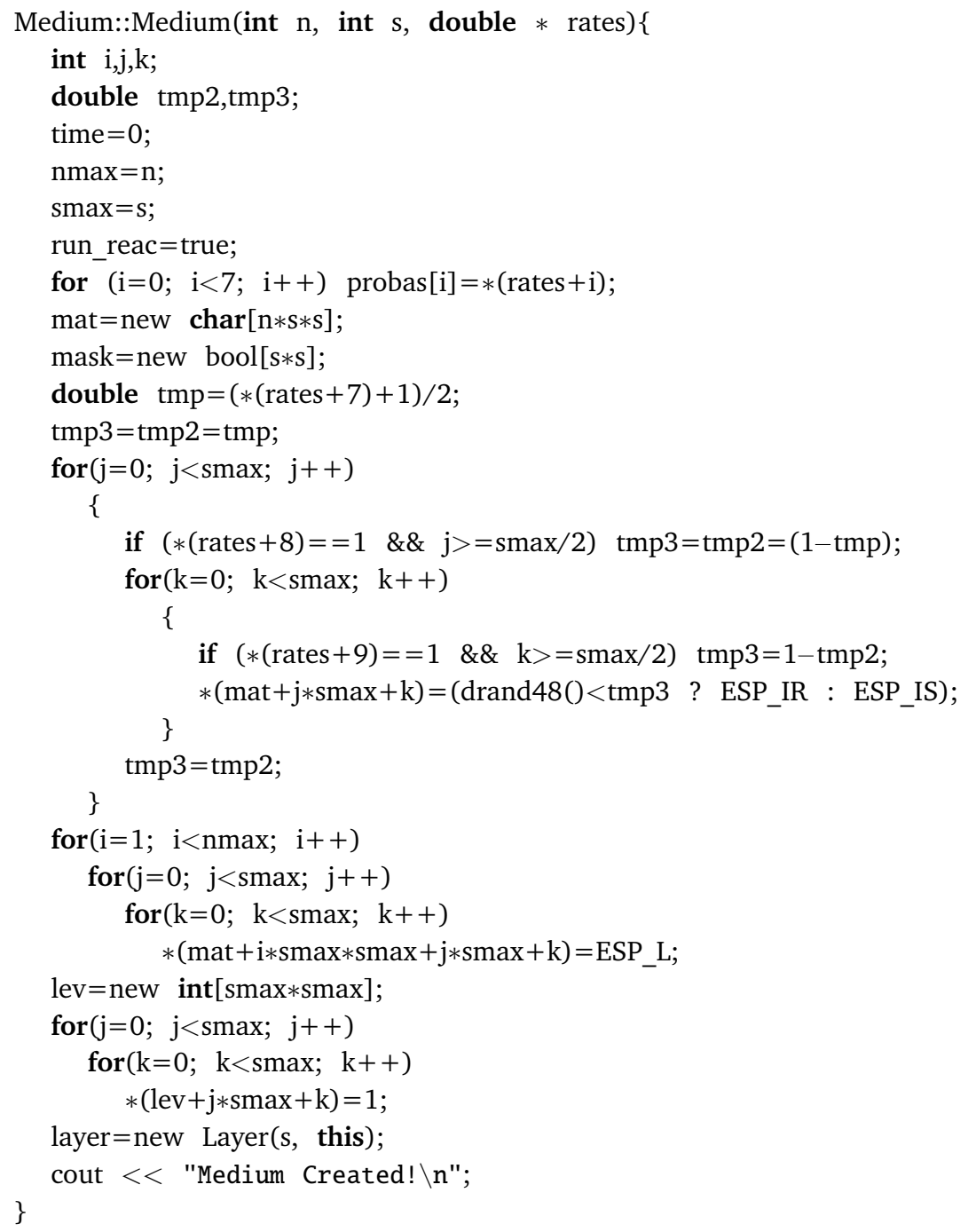

Medium:: Medium()\{

delete[] lev;

delete[] mask;

delete layer;

delete[] mat;

cout $<<$ "Medium Destroyed! \n";

\}

void Medium::make_reac ( Reac* reac )\{

int $\mathrm{n}=$ reac $->\mathrm{n}$;

int $\mathrm{s}=$ reac $->\mathrm{s}$;

int $s 2=$ reac $->s 2$;

$*(\operatorname{mat}+\mathrm{n} * \mathrm{smax} * \operatorname{smax}+\mathrm{s} * \operatorname{smax}+\mathrm{s} 2)=$ reac $->$ next;

if (reac- $>$ type $==$ REAC_20)

$*(\mathrm{lev}+\mathrm{s} * \operatorname{smax}+\mathrm{s} 2)+=1$;

else

$*(\operatorname{mat}+(\mathrm{n}-1) * \operatorname{smax} * \operatorname{smax}+\mathrm{s} * \operatorname{smax}+\mathrm{s} 2)+=2 ;$

if $(*(\mathrm{lev}+\mathrm{s} * \mathrm{smax}+\mathrm{s} 2)==\mathrm{nmax})$

run_reac $=$ false;

else

\{

layer- $>$ update $(*(\mathrm{lev}+\mathrm{s} * \operatorname{smax}+\mathrm{s} 2), \mathrm{s}, \mathrm{s} 2)$;

if $(\mathrm{s}>0)$

layer- $>$ update $(*(\operatorname{lev}+(\mathrm{s}-1) * \operatorname{smax}+\mathrm{s} 2), \mathrm{s}-1, \mathrm{~s} 2)$;

else

layer-> update $(*(\mathrm{lev}+(\operatorname{smax}-1) * \operatorname{smax}+\mathrm{s} 2), \mathrm{smax}-1, \mathrm{~s} 2)$;

if $(s<(\operatorname{smax}-1))$ 


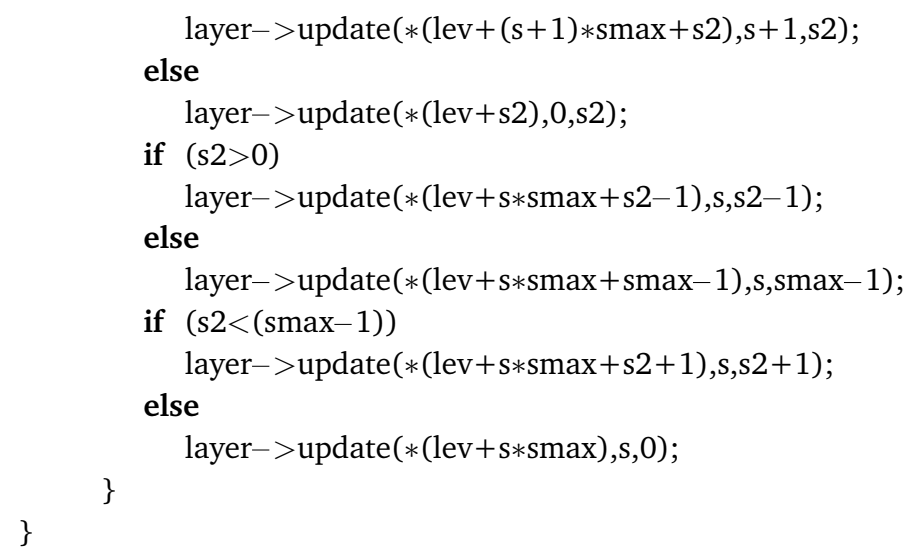

double Medium::process ( int max_step, ofstream** output ) \{ double $\mathrm{t}=0$;

int levelmin,levelmax,tmp;

for (int $\mathrm{i}=0 ; \mathrm{i}<\mathrm{max}$ step \&\& run_reac $; \mathrm{i}++$ )

$\mathrm{t}+=$ layer $->$ process_reac () ;

time $+=\mathrm{t}$;

cout $<<$ "t=" $<<$ time $<<$ " \n";

**output $<<$ "\n";

$* *$ (output +1 ) $<<" \backslash \mathrm{n} "$;

levelmin $=$ levelmax $=*$ lev;

for (int $\mathrm{j}=0 ; \mathrm{j}<\operatorname{smax} ; \mathrm{j}++$ )

\{

for (int $\mathrm{k}=0 ; \mathrm{k}<\mathrm{smax} ; \mathrm{k}++$ )

\{

int level $=*(\mathrm{lev}+\mathrm{j} * \operatorname{smax}+\mathrm{k})$;

if (levelmin $>$ level) levelmin=level;

if (levelmax $<$ level) levelmax=level;

$* *$ output $<<$ level $<<$ " ";

tmp $=($ int $)(*($ mat + level $* \operatorname{smax} * \operatorname{smax}+\mathrm{j} * \mathrm{smax}+\mathrm{k}))$;

if $(\mathrm{tmp}==0) \quad \mathrm{tmp}=($ int $)(*($ mat $+($ level -1$) * \operatorname{smax} * \operatorname{smax}+\mathrm{j} * \operatorname{smax}+\mathrm{k}))$;

$* *$ (output +1$)<<$ tmp $<<"$ ";

\}

**output $<<$ "\n";

$* *$ (output +1$) \quad<<" \backslash \mathrm{n} "$;

\}

**(output +4$)<<$ time $<<" ~ "<<$ levelmin $<<$ " "

$<<$ levelmax $<<"$ " $<<" \backslash \mathrm{n} "$;

return $t$

\}

void Medium::slice_out (ofstream $*$ output $)\{$

for (int $\mathrm{j}=0 ; \mathrm{j}<\mathrm{smax} ; \mathrm{j}++$ )

\{

for (int $\mathrm{i}=0 ; \mathrm{i}<\mathrm{nmax} ; \mathrm{i}++$ )

*output $<<$ (int) $(*(\operatorname{mat}+\mathrm{i} * \operatorname{smax} * \operatorname{smax}+\mathrm{j} * \operatorname{smax}+\operatorname{smax} / 2))<<"$ ";

*output $<<$ "\n";

\}

\}

char Medium::what (int $n$, int $s$, int $s 2)\{$

if $(\mathrm{s}<0) \mathrm{s}+=\mathrm{smax}$;

if $(s 2<0) s 2+=\operatorname{smax}$

if $(\mathrm{n}<0) \mathrm{n}+=$ nmax;

if $(\mathrm{s}>=\operatorname{smax}) \mathrm{s}-=\mathrm{smax}$;

if $(\mathrm{s} 2>=\operatorname{smax}) \mathrm{s} 2-=\mathrm{smax}$;

if $(\mathrm{n}>=\mathrm{nmax}) \mathrm{n}-=\mathrm{nmax}$;

return $*($ mat $+\mathrm{n} * \mathrm{smax} * \mathrm{smax}+\mathrm{s} * \mathrm{smax}+\mathrm{s} 2)$; 
\}

Linked_node $*$ Medium::what_node (int $\mathrm{n}$, int $\mathrm{s}$, int $\mathrm{s} 2$ ) \{

if $(\mathrm{s}<0) \mathrm{s}+=$ smax;

if $(s 2<0) s 2+=\operatorname{smax}$

if $(\mathrm{n}<0) \mathrm{n}+=$ nmax;

if $(\mathrm{s}>=\mathrm{smax}) \mathrm{s}-=$ smax;

if $(s 2>=s \operatorname{sax}) \mathrm{s} 2-=\operatorname{smax}$;

if $(\mathrm{n}>=\mathrm{nmax}) \mathrm{n}-=\mathrm{nmax}$;

Linked node $* \operatorname{tmp}=*($ node $+\mathrm{n} * \operatorname{smax} * \operatorname{smax}+\mathrm{s} * \operatorname{smax}+\mathrm{s} 2)$;

if $(\mathrm{tmp} !=\mathrm{NULL})$

while $($ tmp $->$ next $!=$ NULL $)$

tmp $=$ tmp $->$ next;

return tmp;

\}

double Medium::proba (char type)\{ return probas[(int)type]; \}

void Medium::ee_out (ofstream * output) \{

for (int $\mathrm{i}=0 ; \mathrm{i}<\mathrm{nmax} ; \mathrm{i}++$ )

\{

int $\operatorname{tmp}=0$;

for (int $\mathrm{j}=0 ; \mathrm{j}<\operatorname{smax} ; \mathrm{j}++$ )

for (int $\mathrm{k}=0 ; \mathrm{k}<\operatorname{smax} ; \mathrm{k}++$ )

\{

char $\operatorname{tmp} 2=*(\operatorname{mat}+\mathrm{i} * \operatorname{smax} * \operatorname{smax}+\mathrm{j} * \operatorname{smax}+\mathrm{k})$;

if (tmp2 $!=0)$

tmp $+=((\operatorname{tmp} 2 \% 2==0) \quad ? \quad 1:-1)$;

\}

*output $<$ i $<<$ " " $<$ (double)tmp/(double)(smax*smax) $<<$ "\n";

\}

void Medium::struct_out (ofstream * output) \{

for (int $\mathrm{i}=0 ; \mathrm{i}<\mathrm{nmax} ; \mathrm{i}++$ )

\{

int total $=0$;

int $\operatorname{sum}=0$;

int contact $=0$;

for (int $\mathrm{j}=0 ; \mathrm{j}<\operatorname{smax} ; \mathrm{j}++$ )

for (int $\mathrm{k}=0 ; \mathrm{k}<\operatorname{smax} ; \mathrm{k}++$ )

\{

char current $=$ what $(\mathrm{i}, \mathrm{j}, \mathrm{k})$;

if (current $!=0$ )

\{

current $\%=2$;

sum $+=$ current;

total ++ ;

char rightside $=$ what $(\mathrm{i}, \mathrm{j}+1, \mathrm{k})$;

if (rightside $!=0$ )

\{

rightside $\%=2$;

contact $+=$ (current $!=$ rightside $)$;

\}

else

contact ++ ;

char downside $=$ what $(\mathrm{i}, \mathrm{j}, \mathrm{k}+1)$;

if (downside $!=0$ )

\{

downside $\%=2$;

contact $+=$ (current $!=$ downside $)$; 


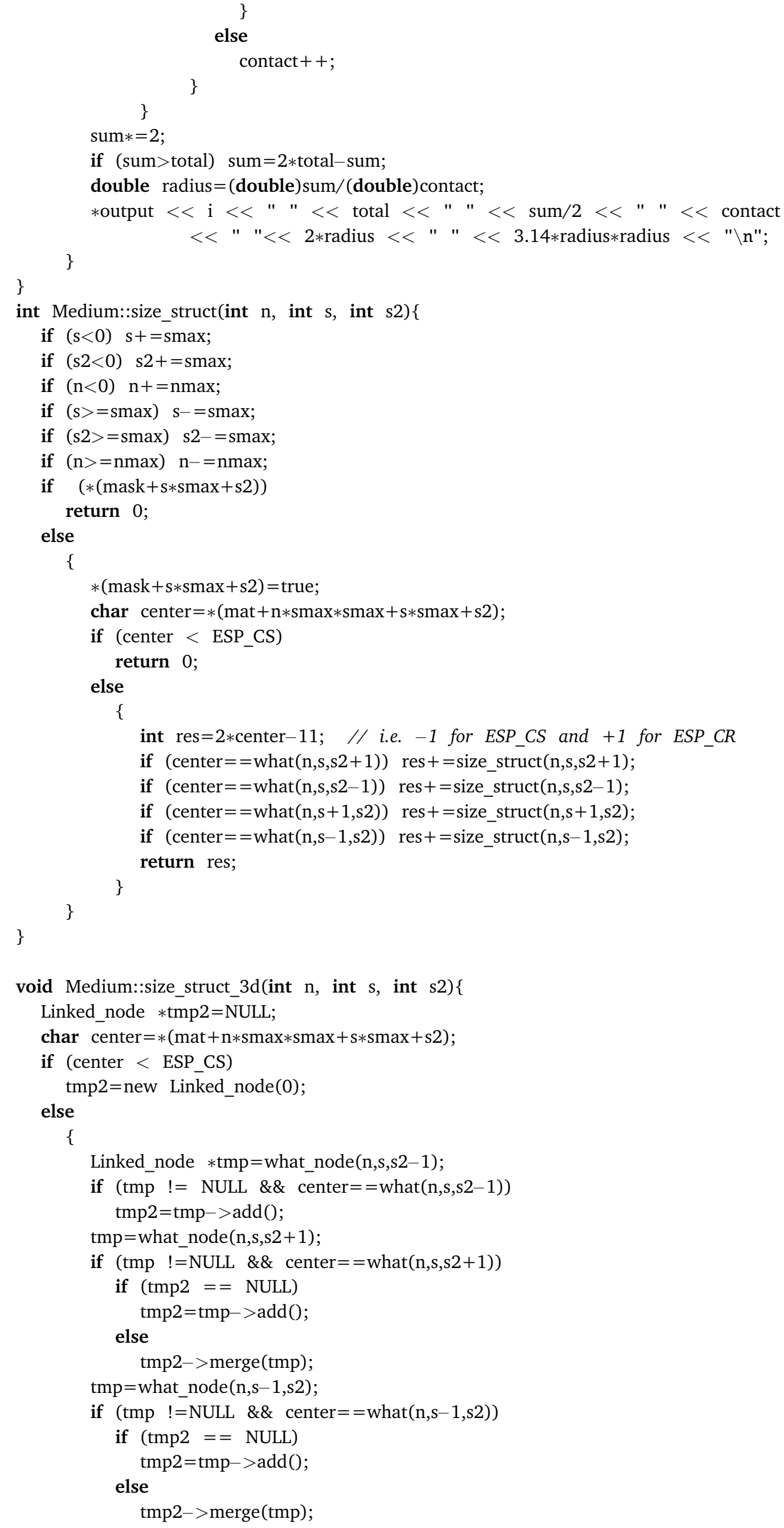




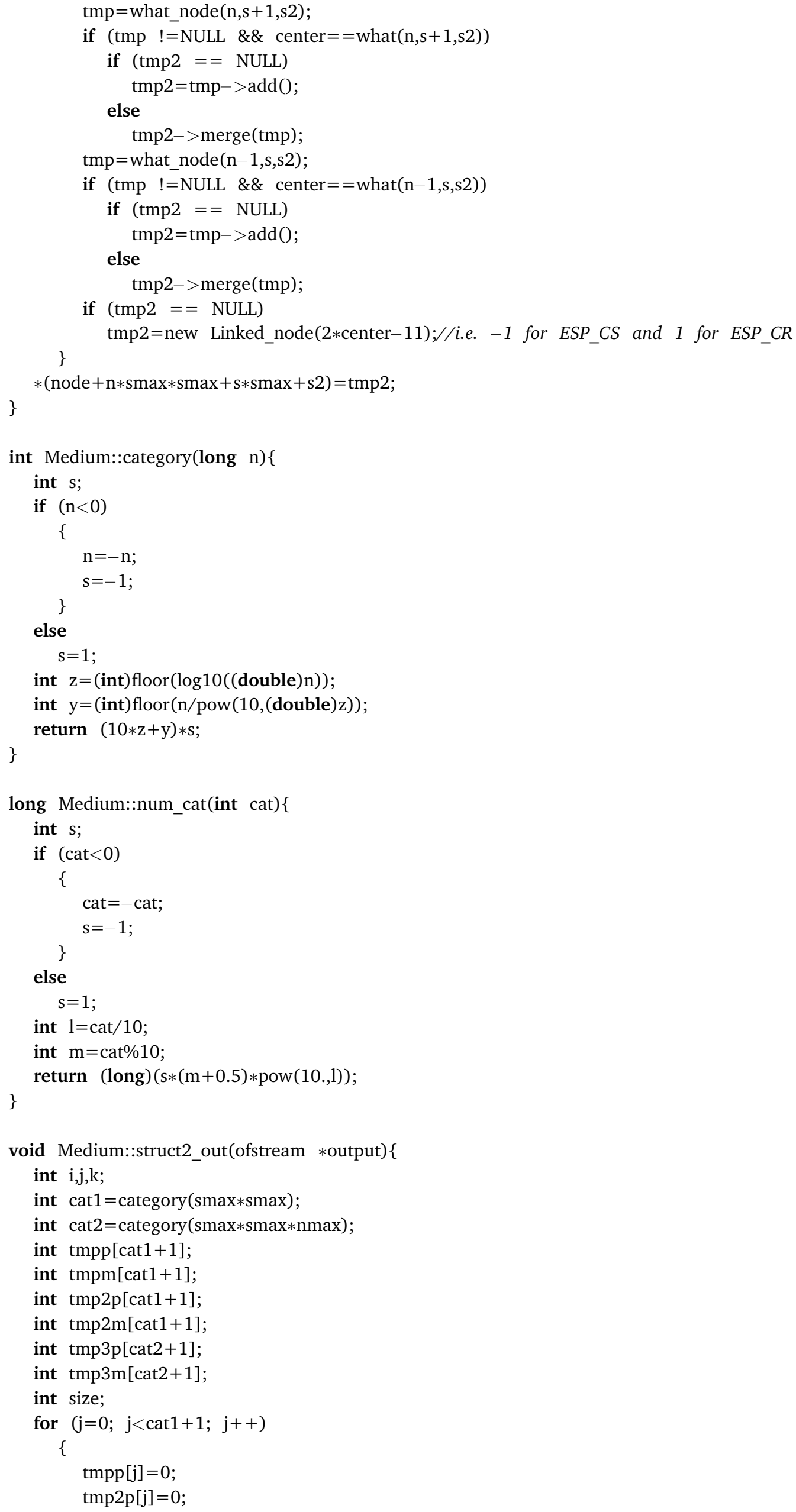




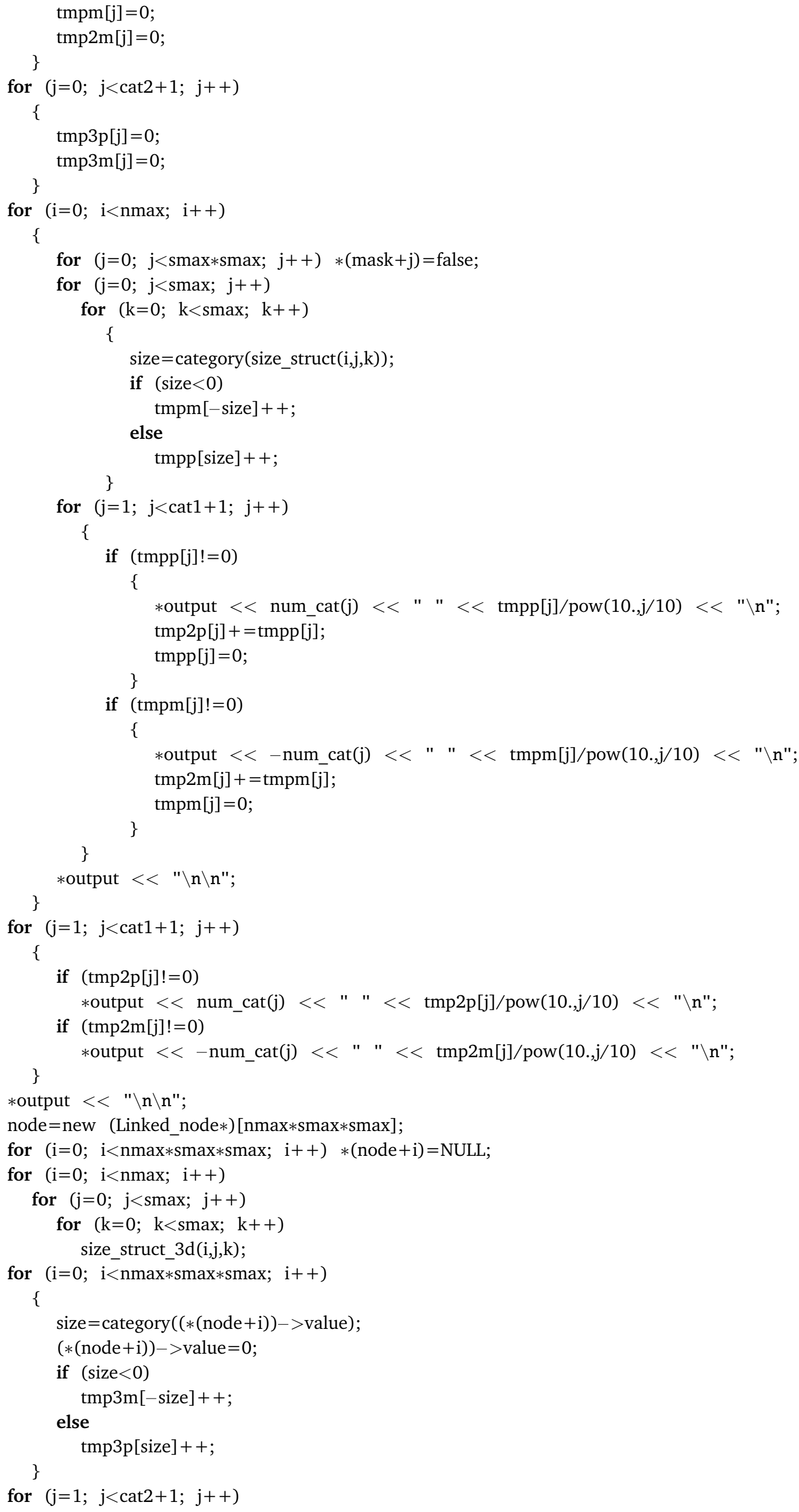




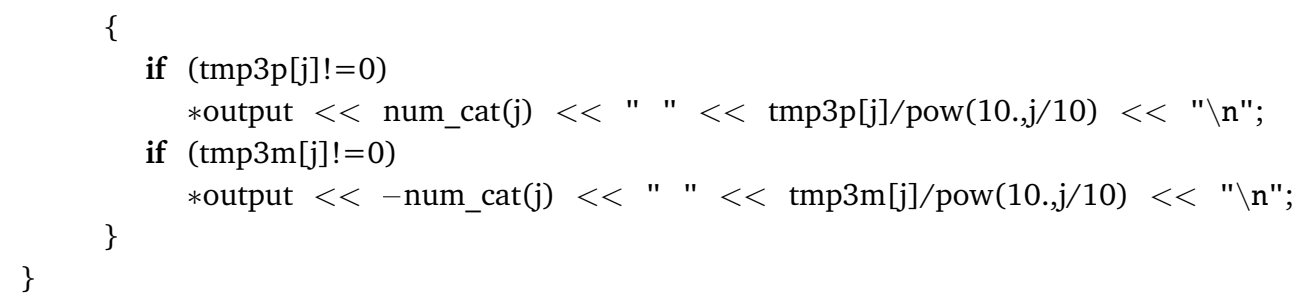

\subsection{Layer and Sublayer Class}

These classes are the link between medium class and event class. An initially more general layer class have been splitted into these two layer and sublayer classes for calculation time optimization purpose. They permit to choose from the medium class which possible reaction (one instance of event class) will occur.

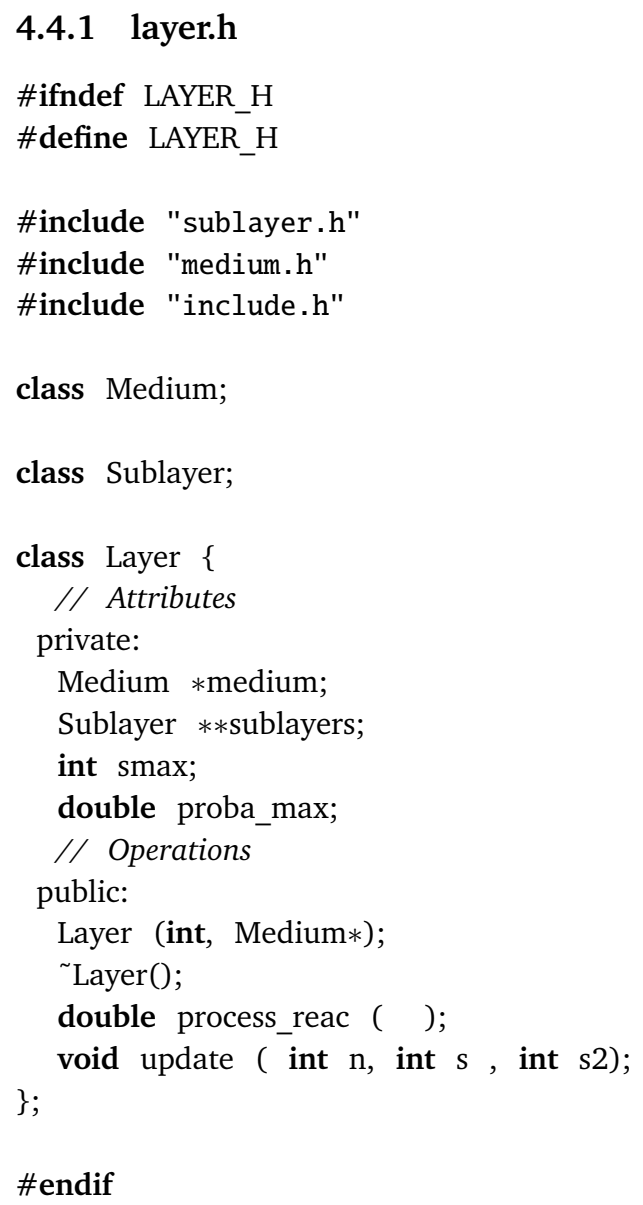

\subsection{2 layer.cpp}

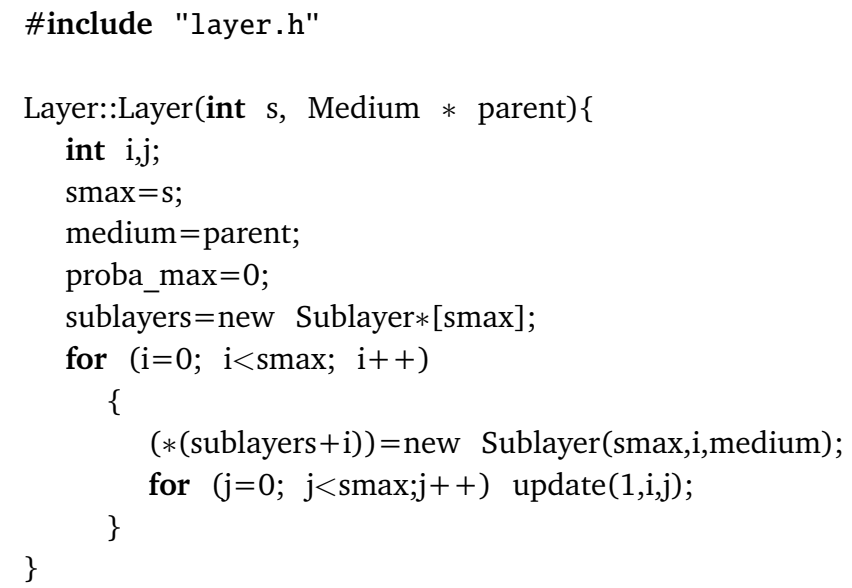




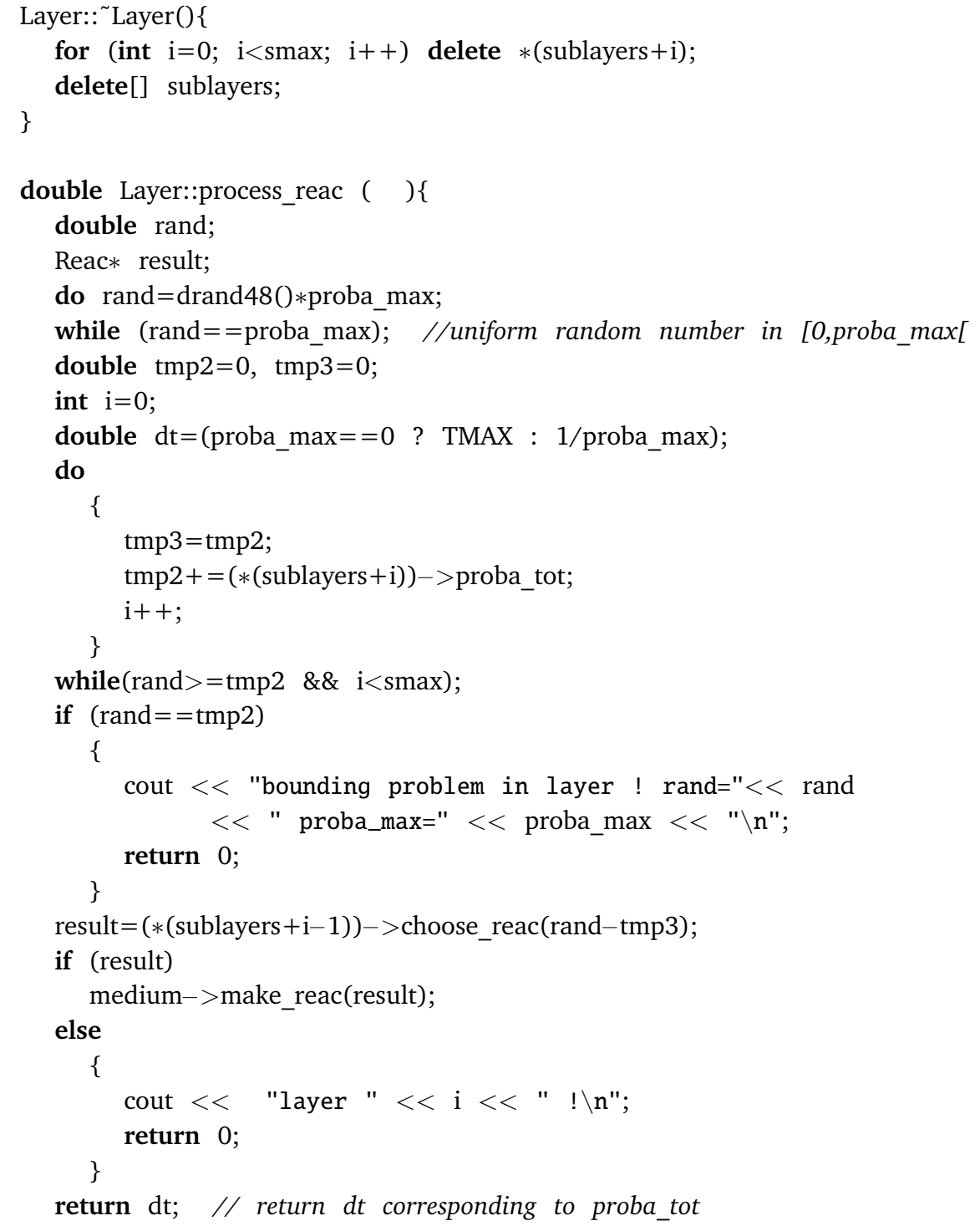

\subsection{3 sublayer.h}

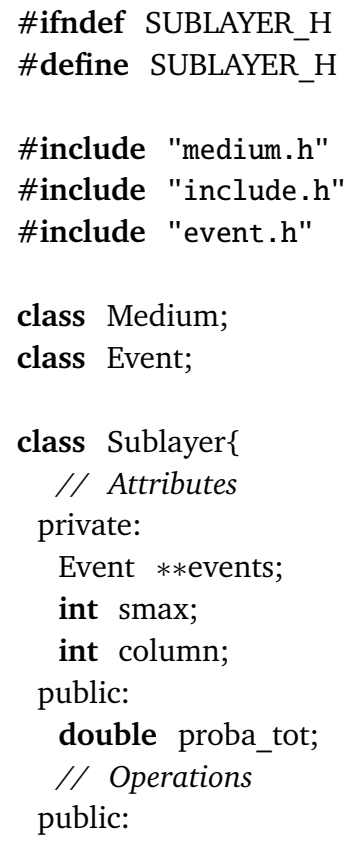


Sublayer (int,int,Medium*);

Sublayer();

Reac* choose_reac (double);

\};

double update ( int $\mathrm{n}$, int $\mathrm{s}$ );

\# endif

\subsection{4 sublayer.cpp}

\#include "sublayer.h"

Sublayer::Sublayer(int s, int pos, Medium *medium)\{

int $\mathrm{i}$;

$\operatorname{smax}=\mathrm{s}$;

column $=$ pos;

proba_tot $=0$;

events $=$ new Event $*[\mathrm{~s}]$;

for $(\mathrm{i}=0 ; \mathrm{i}<$ smax; $\mathrm{i}++) *($ events $+\mathrm{i})=$ new Event(medium);

\}

Sublayer:: Sublayer()\{

for (int $\mathrm{i}=0 ; \mathrm{i}<$ smax; $\mathrm{i}++$ ) delete $*($ events $+\mathrm{i}$ );

//free(events);

delete events;

\}

Reac* Sublayer::choose_reac (double alea) \{

double $\operatorname{tmp} 2=0, \operatorname{tmp} 3=0$;

int $i=0$;

do

\{

tmp3 $=$ tmp 2

tmp $2+=(*($ events $+\mathrm{i}))->$ proba_tot;

$\mathrm{i}++$;

\}

while(alea $>=$ tmp2 \&\& $\mathrm{i}<\operatorname{smax}$ );

if $($ alea $==\operatorname{tmp} 2)$

\{

cout $<<$ "bounding problem in sublayer ! alea=" $<<$ alea $<<$ " proba_max=" $<<$ proba_tot $<<$ "\n";

return NULL;

\}

return $(*($ events $+\mathrm{i}-1))->$ choose_reac(alea-tmp3);

\}

double Sublayer::update ( int $\mathrm{n}$, int $\mathrm{s})\{$

double diff_proba $=(*($ events $+\mathrm{s}))->$ update $(\mathrm{n}$, column,s);

proba_tot $+=$ diff_proba;

return diff_proba;

\}

\subsection{Event Class}

This class instantiate the several possible reactions than can occur in the system, and can perform them when asked through the medium->layer->sublayer chain.

\subsection{1 event.h}

\#ifndef EVENT_H 


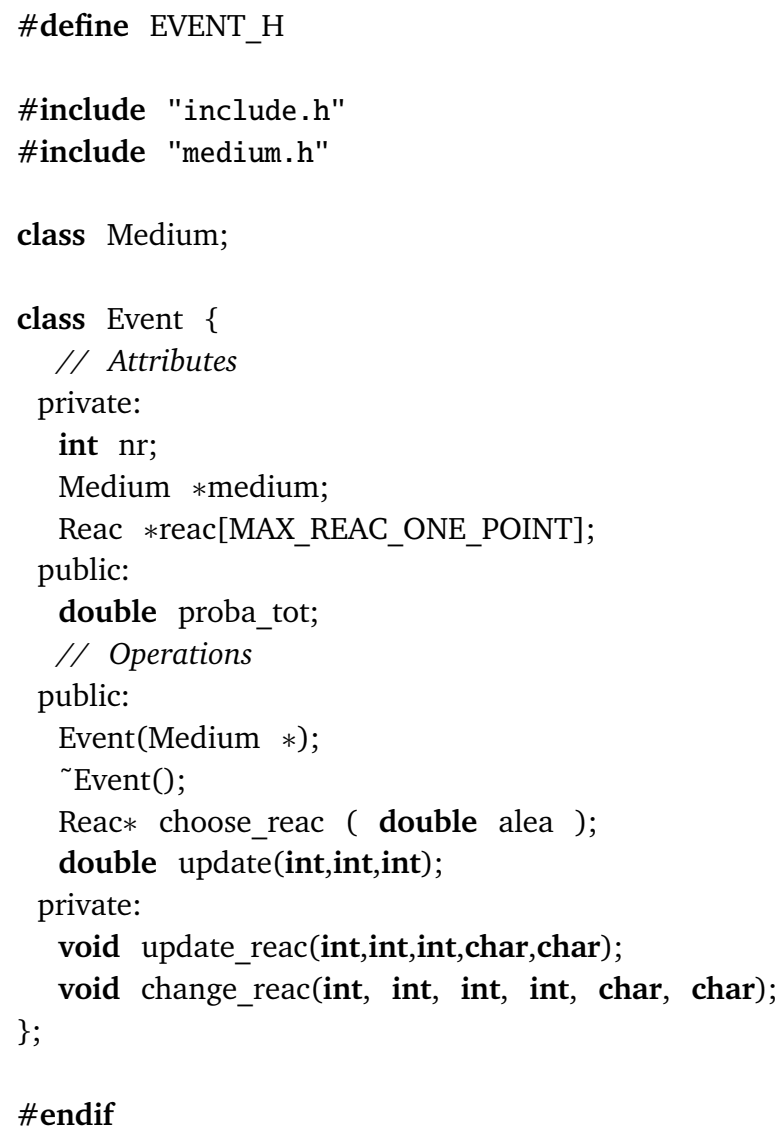

\subsection{2 event.cpp}

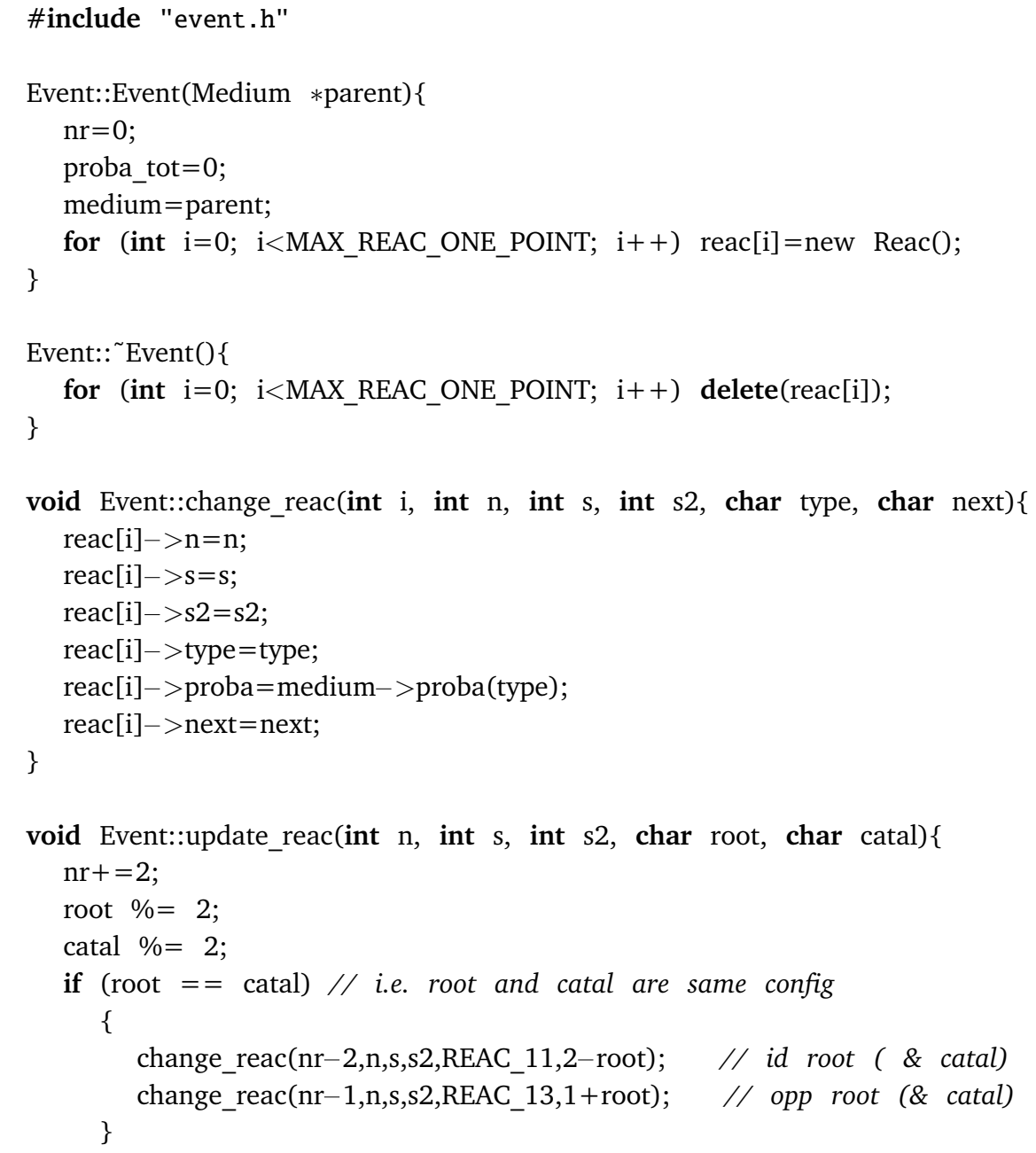




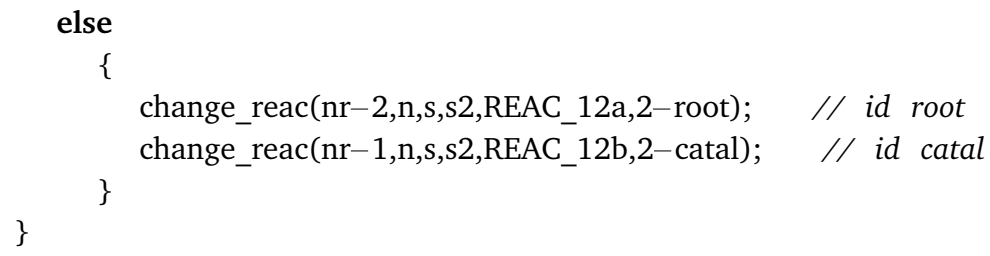

